\title{
Analysis of Bifurcations of Limit Cycles with Lyapunov Exponents and Numerical Normal Forms
}

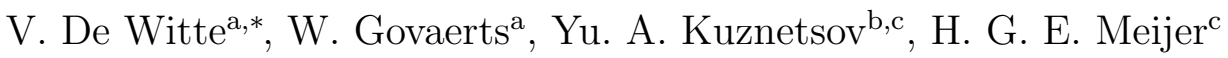 \\ ${ }^{a}$ Department of Applied Mathematics and Computer Science, Ghent University, \\ Krijgslaan 281-S9, B-9000, Gent, Belgium \\ ${ }^{b}$ Department of Mathematics, Utrecht University, Budapestlaan 6, P.O. Box 80010, 3508 \\ TA Utrecht, The Netherlands \\ ${ }^{c}$ Department of Applied Mathematics, University of Twente, 7500 AE Enschede, The \\ Netherlands
}

\begin{abstract}
In this paper we focus on the combination of normal form and Lyapunov exponent computations in the numerical study of the three codim 2 bifurcations of limit cycles with dimension of the center manifold equal to 4 or to 5 in generic autonomous ODEs. The normal form formulas are independent of the dimension of the phase space and involve solutions of certain linear boundary-value problems. The formulas allow one to distinguish between the complicated bifurcation scenarios which can happen near these codim 2 bifurcations, where 3-tori and 4-tori can be present. We apply our techniques to the study of a known laser model, a novel model from population biology, and a model of mechanical vibrations. These models exhibit Limit Point-Neimark-Sacker, Period-Doubling-Neimark-Sacker, and double Neimark-Sacker bifurcations. Lyapunov exponents are computed to numerically confirm the results of the normal form analysis, in particular with respect to the existence of stable invariant tori of various dimensions. Conversely, the normal forms are essential to understand the significance of the Lyapunov exponents.
\end{abstract}

Keywords: Fold-Neimark-Sacker, Flip-Neimark-Sacker, double Neimark-Sacker, 3-torus, 4-torus, normal form

\footnotetext{
*Corresponding author

Email addresses: Virginie.DeWitte@UGent.be (V. De Witte), Willy.Govaerts@UGent.be (W. Govaerts), I.A.Kouznetsov@uu.nl (Yu. A. Kuznetsov), h.g.e.meijer@utwente.nl (H. G. E. Meijer)
} 
2000 MSC: 34C20, 37G15, 37M20, 65L07

\section{Introduction}

Consider a smooth system of ODEs

$$
\dot{x}=f(x, p), \quad x \in \mathbb{R}^{n},
$$

smoothly depending on a parameter vector $p \in \mathbb{R}^{m}$. Typically, the dynamics of such systems show qualitative transitions, i.e. bifurcations, upon variation of a parameter. It is hard to use simulations to characterize such transitions correctly and efficiently. Numerical continuation software such as AUTO [1] or MATCONT $[2,3]$ may be used to track bifurcations from a stable equilibrium to a periodic oscillation by a Hopf bifurcation and even the appearance of (un)stable invariant tori with multi-frequency oscillations by a secondary Hopf, or Neimark-Sacker bifurcation. Bifurcations of these invariant tori $\mathbb{T}^{m \geq 2}$ into other tori or chaos, however, are out of reach of the standard numerical analysis.

One possibility to study bifurcations of tori - if they are stable - is to compute Lyapunov exponents. The dimension of the torus for a given parameter value then equals the number of exponents equal to zero. Varying one parameter one can observe that exponents become zero and this indicates a bifurcation. The exact nature of the bifurcation is however obscured from this analysis and should be elucidated with additional means. Yet, in many cases, bifurcations of tori first emerge from codim 2 bifurcations of limit cycles. Specifically, these codim 2 bifurcations are points in the parameter plane where one Neimark-Sacker bifurcation curve intersects a Limit Point of cycles, a Period-Doubling or another Neimark-Sacker bifurcation curve. The intersections produce LPNS, PDNS, or NSNS bifurcations, respectively. This paper focuses on these bifurcations, occuring in generic systems (1) when $m \geq 2$ and $n$ is sufficiently large. The bifurcations are well understood theoretically with Poincaré maps and the corresponding normal forms $[4,5,6,7,8,9,10]$. The results of the analyis of the normal form for these codim 2 bifurcations can be used to verify nondegeneracy conditions and classify the bifurcation structure. Hence, we need an algorithm for the numerical computation of the coefficients of each critical normal form to enable this analysis.

There is a straightforward approach to obtain the critical normal forms of the codim 2 bifurcations of the limit cycle. In the Poincaré map, the 
limit cycle corresponds to a fixed point and one can use techniques developed for maps to obtain the critical normal form $[8,9]$. However, in this case partial derivatives of the map up to order $k$, most often $k=3$, sometimes $k=5$, are needed. Alternatively one could integrate the variational equations [11] or use automatic differentiation $[12,13]$ to obtain the derivatives of the Poincaré map. All these methods, however, have two drawbacks that make them less (time) efficient. First, these are shooting methods that are slower when the system is very sensitive to perturbations. Second, the full Poincaré map is computed while only certain expressions are needed for the normalization. There is an alternative technique that is more suitable in the context of numerical continuation of periodic orbits using collocation as the whole periodic orbit is available. It uses periodic normalization $[14,15]$ and has been applied to codim 1 bifurcations of limit cycles and implemented in MATCONT [16]. This technique uses orthogonal collocation in the solution of boundary value problems and does not need to compute the corresponding tensors.

Recently, we have extended this algorithm to codim 2 bifurcations of limit cycles with center manifold dimension at most 3 [17]. Here we consider the three remaining and most difficult cases, LPNS, PDNS, and NSNS, that are characterized by a center manifold of the critical cycle of dimension 4 or 5. These three cases always involve a - possibly unstable - two-dimensional torus $\mathbb{T}^{2}$ and in many cases also a 3 -dimensional torus $\mathbb{T}^{3}$ and a 4-dimensional torus $\mathbb{T}^{4}$.

We have implemented our algorithm in the numerical continuation toolbox MATCONT which automatically invokes the algorithm whenever the corresponding bifurcation is detected. Hence, any user is able to use it and take advantage of the automated normal form analysis. Here we document precisely what our algorithm does. First, our aim is to derive coefficients of a periodic critical normal form. We present these normal forms in Section 2 using (contrary to $[16,17]$ ) the original Iooss [14] representation. Remark that these normal forms are closely related to the normal forms for the Zero-Hopf and Hopf-Hopf bifurcations of equilibria. We discuss the correspondence and the interpretation of the bifurcation diagrams of the generic unfoldings for the LPNS, PDNS, and NSNS bifurcations. Next, we present the formulas to compute the critical normal form coefficients in Section 3. We extensively discuss the LPNS case but omit details in the PDNS and NSNS cases (the complete discussion can be found in [18]). Here we also comment on the implementation which is similar to [17]. Finally in Section 4, we consider 
several examples that involve tori bifurcations: a laser model, a model from population biology, and one for mechanical vibrations. In these models we find and analyze the three codim 2 bifurcations that we focus on. We compute the critical normal form coefficients using our algorithm to predict the bifurcation diagram near each of these codim 2 points. Next we corroborate the predictions using Lyapunov exponents. In fact, we argue that the classification from the critical normal form guides the correct interpretation of the Lyapunov exponents.

\section{Normal forms on the center manifold and their bifurcations}

Write (1) at the critical parameter values as

$$
\dot{u}=F(u)
$$

and suppose that there is a limit cycle $\Gamma$ corresponding to a periodic solution $u_{0}(t)=u_{0}(t+T)$, where $T>0$ is its (minimal) period. Expand $F\left(u_{0}(t)+v\right)$ into the Taylor series

$$
\begin{aligned}
F\left(u_{0}(t)+v\right)= & F\left(u_{0}(t)\right)+A(t) v+\frac{1}{2} B(t ; v, v)+\frac{1}{3 !} C(t ; v, v, v)+ \\
& \frac{1}{4 !} D(t ; v, v, v, v)+\frac{1}{5 !} E(t ; v, v, v, v, v)+O\left(\|v\|^{6}\right),
\end{aligned}
$$

where $A(t)=F_{u}\left(u_{0}(t)\right)$ and

$$
B\left(t ; v_{1}, v_{2}\right)=F_{u u}\left(u_{0}(t)\right)\left[v_{1}, v_{2}\right], \quad C\left(t ; v_{1}, v_{2}, v_{3}\right)=F_{\text {uuu }}\left(u_{0}(t)\right)\left[v_{1}, v_{2}, v_{3}\right],
$$

etc. The matrix $A$ and the multilinear forms $B, C, D$, and $E$ are periodic in $t$ with period $T$ but this dependence will often not be indicated explicitly.

Consider the initial-value problem for the fundamental matrix solution $Y(t)$, namely,

$$
\frac{d Y}{d t}=A(t) Y, \quad Y(0)=I_{n},
$$

where $I_{n}$ is the $n \times n$ identity matrix. The eigenvalues of the monodromy matrix $M=Y(T)$ are called (Floquet) multipliers of the limit cycle. The multipliers with $|\mu|=1$ are called critical. There is always a "trivial" critical multiplier $\mu_{n}=1$. We denote the total number of critical multipliers by $n_{c}$ and assume that the limit cycle is non-hyperbolic, i.e. $n_{c}>1$. In this case, 
there exists an invariant $n_{c^{-}}$dimensional critical center manifold $W^{c}(\Gamma) \subset \mathbb{R}^{n}$ near $\Gamma^{1}$.

\subsection{Critical normal forms}

It is well known $[19,7]$ that in generic two-parameter systems (1) only eleven codim 2 local bifurcations of limit cycles occur. To describe the normal forms of $(2)$ on the critical center manifold $W^{c}(\Gamma)$ for these codim 2 cases, we parameterize $W^{c}(\Gamma)$ near $\Gamma$ by $\left(n_{c}-1\right)$ transverse coordinates and $\tau \in[0, k T]$ for $k \in\{1,2\}$, depending on the bifurcation. The 8 cases with $n_{c} \leq 3$ were treated in [17]. Based on [14] we showed in Appendix A in [18] that the restriction of (2) to the corresponding critical center manifold $W^{c}(\Gamma)$ with $n_{c}=4$ or $n_{c}=5$ will take one of the following Iooss normal forms.

\subsection{1. $L P N S$}

The Limit Point - Neimark-Sacker bifurcation occurs when the trivial critical multiplier $\mu_{n}=1$ corresponds to a two-dimensional Jordan block and there are only two more critical simple multipliers $\mu_{1,2}=e^{ \pm i \theta}$ with $\theta \neq \frac{2 \pi}{j}$, for $j=1,2,3,4$. The four-dimensional Iooss normal form at the LPNS bifurcation is derived in Appendix A.1.1 in [18] and can be written as

$$
\left\{\begin{array}{l}
\frac{d \tau}{d t}=1-\xi_{1}+\alpha_{200} \xi_{1}^{2}+\alpha_{011}\left|\xi_{2}\right|^{2}+\alpha_{300} \xi_{1}^{3}+\alpha_{111} \xi_{1}\left|\xi_{2}\right|^{2}+\ldots \\
\frac{d \xi_{1}}{d \tau}=a_{200} \xi_{1}^{2}+a_{011}\left|\xi_{2}\right|^{2}+a_{300} \xi_{1}^{3}+a_{111} \xi_{1}\left|\xi_{2}\right|^{2}+\ldots \\
\frac{d \xi_{2}}{d \tau}=i \omega \xi_{2}+b_{110} \xi_{1} \xi_{2}+b_{210} \xi_{1}^{2} \xi_{2}+b_{021} \xi_{2}\left|\xi_{2}\right|^{2}+\ldots
\end{array}\right.
$$

where $\tau \in[0, T], \omega=\theta / T, \xi_{1}$ is a real coordinate and $\xi_{2}$ is a complex coordinate on $W^{c}(\Gamma)$ transverse to $\Gamma, \alpha_{i j k}, a_{i j k} \in \mathbb{R}, b_{i j k} \in \mathbb{C}$, and the dots denote the $O\left(\left\|\xi^{4}\right\|\right)$-terms, which are $T$-periodic in $\tau$. The equations (4) implicitly describe motions on the 4-dimensional invariant manifold $W^{c}(\Gamma)$ with one cyclic coordinate $\tau$.

\subsection{2. $P D N S$}

The Period-Doubling - Neimark-Sacker bifurcation occurs when the trivial critical multiplier $\mu_{n}=1$ is simple and there are only three more critical

\footnotetext{
${ }^{1}$ This manifold should not be confused with the $\left(n_{c}-1\right)$-dimensional center manifold of the corresponding Poincaré map.
} 
simple multipliers, namely -1 and $\mu_{1,2}=e^{ \pm i \theta}$ with $\theta \neq \frac{2 \pi}{j}$, for $j=1,2,3,4$. The four-dimensional Iooss normal form at the PDNS bifurcation is derived in Appendix A.1.2 in [18] and can be written as

$$
\left\{\begin{aligned}
\frac{d \tau}{d t}= & 1+\alpha_{200} \xi_{1}^{2}+\alpha_{011}\left|\xi_{2}\right|^{2}+\alpha_{400} \xi_{1}^{4}+\alpha_{022}\left|\xi_{2}\right|^{4}+\alpha_{211} \xi_{1}^{2}\left|\xi_{2}\right|^{2}+\ldots \\
\frac{d \xi_{1}}{d \tau}= & a_{300} \xi_{1}^{3}+a_{111} \xi_{1}\left|\xi_{2}\right|^{2}+a_{500} \xi_{1}^{5}+a_{122} \xi_{1}\left|\xi_{2}\right|^{4}+a_{311} \xi_{1}^{3}\left|\xi_{2}\right|^{2}+\ldots \\
\frac{d \xi_{2}}{d \tau}= & i \omega \xi_{2}+b_{210} \xi_{1}^{2} \xi_{2}+b_{021} \xi_{2}\left|\xi_{2}\right|^{2}+b_{410} \xi_{1}^{4} \xi_{2}+b_{221} \xi_{1}^{2} \xi_{2}\left|\xi_{2}\right|^{2} \\
& +b_{032} \xi_{2}\left|\xi_{2}\right|^{4}+\ldots
\end{aligned}\right.
$$

where $\tau \in[0,2 T], \omega=\theta / T, \xi_{1}$ is a real coordinate and $\xi_{2}$ is a complex coordinate on $W^{c}(\Gamma)$ transverse to $\Gamma, \alpha_{i j k}, a_{i j k} \in \mathbb{R}, b_{i j k} \in \mathbb{C}$, and the dots denote the $O\left(\left\|\xi^{6}\right\|\right)$-terms, which are $2 T$-periodic in $\tau$. The equations (5) implicitly describe motions on the 4-dimensional invariant manifold $W^{c}(\Gamma)$ that is doubly covered by the selected coordinates.

\subsubsection{NSNS}

The double Neimark-Sacker bifurcation occurs when the trivial critical multiplier $\mu_{n}=1$ is simple and there are only four more critical simple multipliers $\mu_{1,4}=e^{ \pm i \theta_{1}}$ and $\mu_{2,3}=e^{ \pm i \theta_{2}}$ with $\theta_{1,2} \neq \frac{2 \pi}{j}$, for $j=1,2,3,4,5,6$ and $l \theta_{1} \neq j \theta_{2}$ for $l, j \in \mathbb{Z}$ with $l+j \leq 4$ (see [9]). The five-dimensional periodic normal form at the NSNS bifurcation is derived in Appendix A.1.3 in [18] and can be written as

$$
\left\{\begin{aligned}
\frac{d \tau}{d t}= & 1+\alpha_{1100}\left|\xi_{1}\right|^{2}+\alpha_{0011}\left|\xi_{2}\right|^{2}+\alpha_{2200}\left|\xi_{1}\right|^{4}+\alpha_{0022}\left|\xi_{2}\right|^{4} \\
& +\alpha_{1111}\left|\xi_{1}\right|^{2}\left|\xi_{2}\right|^{2}+\ldots \\
\frac{d \xi_{1}}{d \tau}= & i \omega_{1} \xi_{1}+a_{2100} \xi_{1}\left|\xi_{1}\right|^{2}+a_{1011} \xi_{1}\left|\xi_{2}\right|^{2}+a_{3200} \xi_{1}\left|\xi_{1}\right|^{4} \\
& +a_{1022} \xi_{1}\left|\xi_{2}\right|^{4}+a_{2111} \xi_{1}\left|\xi_{1}\right|^{2}\left|\xi_{2}\right|^{2}+\ldots \\
\frac{d \xi_{2}}{d \tau}= & i \omega_{2} \xi_{2}+b_{0021} \xi_{2}\left|\xi_{2}\right|^{2}+b_{1110} \xi_{2}\left|\xi_{1}\right|^{2}+b_{0032} \xi_{2}\left|\xi_{2}\right|^{4} \\
& +b_{2210} \xi_{2}\left|\xi_{1}\right|^{4}+b_{1121} \xi_{2}\left|\xi_{1}\right|^{2}\left|\xi_{2}\right|^{2}+\ldots,
\end{aligned}\right.
$$

where $\tau \in[0, T], \omega_{1,2}=\theta_{1,2} / T, \xi_{1}$ and $\xi_{2}$ are complex coordinates on $W^{c}(\Gamma)$ transverse to $\Gamma, \alpha_{i j k l} \in \mathbb{R}, a_{i j k l}, b_{i j k l} \in \mathbb{C}$, and the dots denote the $O\left(\left\|\xi^{6}\right\|\right)$ terms, which are $T$-periodic in $\tau$. The equations (6) implicitly describe motions on a 5 -dimensional manifold with one cyclic coordinate $\tau$. 


\subsection{Generic unfoldings of the critical normal forms}

Here we describe how the coefficients of the critical normal forms can be used to predict bifurcations of the phase portraits near the critical limit cycles for nearby parameter values. We introduce certain quantities - computable in terms of these coeffcients - that are reported in the MATCONT output and used to distinguish between various bifurcation scenarios in examples in Section 4.

In generic two-parameter systems (1) the considered bifurcations occur at isolated parameter values. By translating the origin of the parameter plane to one of such points, we can consider an unfolding of the corresponding bifurcation and study its canonical local bifurcation diagram for nearby parameter values. It is well known that the critical center manifold $W^{c}(\Gamma)$ can be smoothly continued w.r.t. $p$ in a neighborhood of the bifurcation point, so that the restriction of (1) to this manifold can be studied. Choosing appropriate coordinates $(\xi, \tau)$ on this parameter-dependent invariant manifold, one can transform the restricted system into a parameter-dependent normal form in which $\frac{d \xi}{d \tau}$ has a $\tau$-independent principal part and higher-order terms which are $k T$-periodic in $\tau$ with $k=1$ for LPNS and NSNS and $k=2$ for PDNS. Below we describe bifurcations of these principal parts, i.e., the truncated parameter-dependent autonomous normal forms. Since the dynamics is determined by the $\xi$-equations, we first focus on their bifurcations and then interpret appearing bifurcation diagrams for the original system (1). The new unfolding parameters will be denoted by $\left(\beta_{1}, \beta_{2}\right)$.

\subsection{1. $L P N S$}

Generically, a two-parameter unfolding of (1) near this bifurcation restricted to the center manifold is smoothly orbitally equivalent (with possible time reversal) to a system in which the equations for the transverse coordinates have the form

$$
\left\{\begin{array}{l}
\frac{d \xi}{d \tau}=\beta_{1}+\xi^{2}+s|\zeta|^{2}+O\left(\|(\xi, \zeta, \bar{\zeta})\|^{4}\right), \\
\frac{d \zeta}{d \tau}=\left(\beta_{2}+i \omega_{1}\right) \zeta+(\theta+i \vartheta) \xi \zeta+\xi^{2} \zeta+O\left(\|(\xi, \zeta, \bar{\zeta})\|^{4}\right)
\end{array}\right.
$$

where the $O$-terms are still $T$-periodic in $\tau$. This system is similar to the normal form for the Zero-Hopf bifurcation of equilibria (cf. Theorem 8.6 on page 338 in [7]). In Figure 1 the four possible bifurcation diagrams of the 
amplitude system (with $\zeta=\rho e^{i \varphi}$ ) for (7) without the $O$-terms,

$$
\left\{\begin{array}{l}
\dot{\xi}=\beta_{1}+\xi^{2}+s \rho^{2}, \\
\dot{\rho}=\rho\left(\beta_{2}+\theta \xi+\xi^{2}\right),
\end{array}\right.
$$

are reported depending on the sign of the normal form coefficients $s$ and $\theta$. Note that these unfoldings can also be found in [7]. Here and in what follows a dot means the derivative w.r.t. $\tau$.

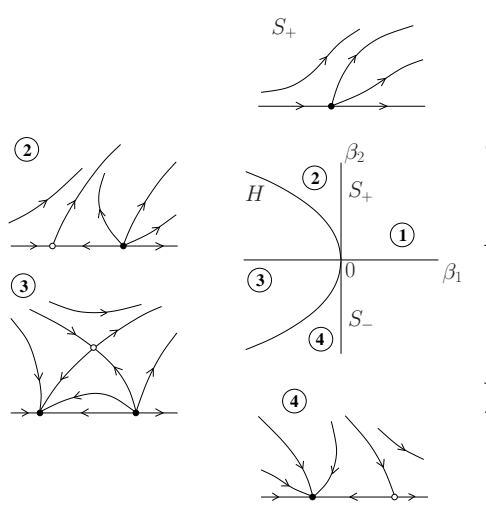

(a) $s=1, \theta>0$

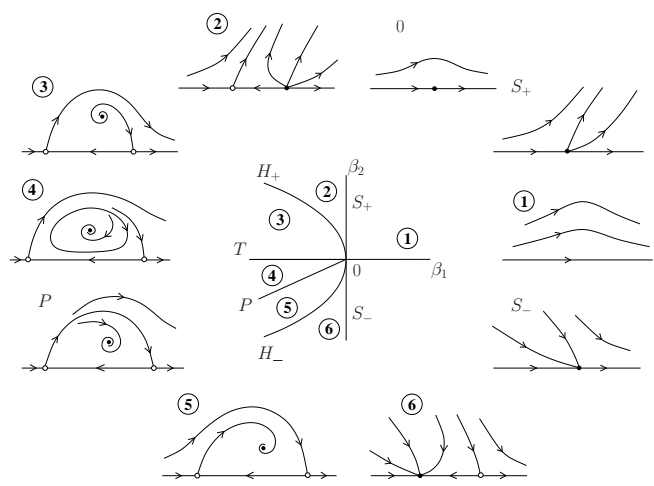

(c) $s=1, \theta<0$
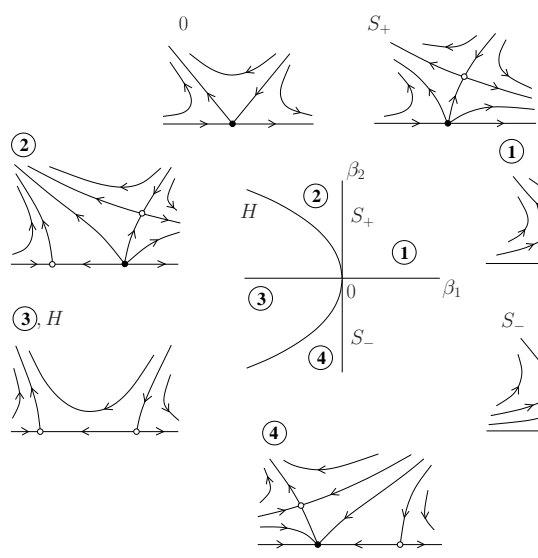

(b) $s=-1, \theta<0$

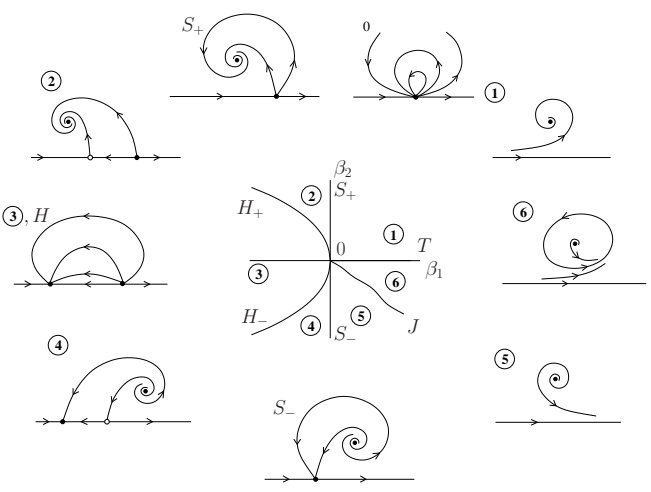

(d) $s=-1, \theta>0$

Figure 1: Bifurcation diagrams of the truncated amplitude system (8) for the LPNS bifurcation.

Let us now discuss the interpretation of the phase portraits in the $(\xi, \rho)$ plane of the truncated amplitude system in the context of the bifurcating limit cycle. The fixed points or limit cycles have additional dimensions from 
the phases of the periodic orbit itself plus the phases ignored in the reduction to the amplitude system. We note that in the amplitude system the vertical $(\rho)$ direction always corresponds to a Neimark-Sacker bifurcation, but that the horizontal $(\xi)$ component of the phase space has a different meaning. For LPNS, equilibria on the horizontal axis correspond to limit cycles. Equilibria off the horizontal axis correspond to invariant $2 \mathrm{D}$ tori $\mathbb{T}^{2}$ and the periodic orbit for (8) which exists if $s \theta<0$ corresponds to an invariant $3 \mathrm{D}$ torus $\mathbb{T}^{3}$ for (1).

The critical values of $s$ and $\theta$ can be expressed in terms of the coeffcients of (4) as

$$
s=\operatorname{sign}\left(a_{200} a_{011}\right), \quad \theta=\frac{\Re\left(b_{110}\right)}{a_{200}} .
$$

These values determine the bifurcation scenario. For $s \theta<0$, a 3 -torus appears in the unfolding via a Neimark-Sacker bifurcation. The stability of this torus is determined by the third order terms in (4). Indeed, the sign of the corresponding first Lyapunov coefficient $l_{1}$ for the Hopf bifurcation in (8) is opposite to that of $\theta$ but the 'time' in (7) is rescaled with factor

$$
E=\Re\left(b_{210}+b_{110}\left(\frac{\Re\left(b_{021}\right)}{a_{011}}-\frac{3 a_{300}}{2 a_{200}}+\frac{a_{111}}{2 a_{011}}\right)-\frac{b_{021} a_{200}}{a_{011}}\right) .
$$

(see page 337 in [7]). If $E \cdot l_{1}<0$, an unstable 3 -torus appears, if $E \cdot l_{1}>0$, the 3 -torus is stable. The output given by matcont is $(s, \theta, E)^{2}$.

Note that Figure 1 presents bifurcations of the truncated system (7) that only approximates the full normalized unfolding. In particular, the orbit structure on the invariant tori can differ from that for the approximating system due to phase locking. Moreover, the destruction of $\mathbb{T}^{3}$ via a heteroclinic bifurcation in case (c) of Figure 1 becomes a complicated sequence of global bifurcations involving stable and unstable invariant sets of cycles and tori. All these bifurcations, however, occur in the exponentially-small parameter wedge near a heteroclinic bifurcation curve. For detailed discussions of the effects of the truncation, also in the two other cases, we refer to $[8,10]$ and references therein.

\subsection{2. $P D N S$}

Generically, a two-parameter unfolding of (1) near this bifurcation restricted to the center manifold is smoothly orbitally equivalent to a system

\footnotetext{
${ }^{2}$ Remark that $E=\mathrm{NaN}$ is reported when terms up to only second order are computed.
} 
in which the equations for the transverse coordinates have the form

$$
\left\{\begin{aligned}
\dot{v}_{1}= & \mu_{1} v_{1}+P_{11} v_{1}^{3}+P_{12} v_{1}\left|v_{2}\right|^{2}+S_{1} v_{1}\left|v_{2}\right|^{4}+O\left(\left\|\left(v_{1}, v_{2}, \bar{v}_{2}\right)\right\|^{6}\right) \\
\dot{v}_{2}= & \left(\mu_{2}+i \omega_{2}\right) v_{2}+P_{21} v_{1}^{2} v_{2}+P_{22} v_{2}\left|v_{2}\right|^{2}+S_{2} v_{1}^{4} v_{2}+i R_{2} v_{2}\left|v_{2}\right|^{4} \\
& +O\left(\left\|\left(v_{1}, v_{2}, \bar{v}_{2}\right)\right\|^{6}\right)
\end{aligned}\right.
$$

where the $O$-terms are still $T$-periodic in $\tau$. This system is similar to one of the normal forms for the Hopf-Hopf bifurcations of equilibria (cf. Lemma 8.14 on page 354 in [7]).

The amplitude system for (9) without the $O$-terms is

$$
\left\{\begin{array}{l}
\dot{r}_{1}=r_{1}\left(\mu_{1}+p_{11} r_{1}^{2}+p_{12} r_{2}^{2}+s_{1} r_{2}^{4}\right) \\
\dot{r}_{2}=r_{1}\left(\mu_{2}+p_{21} r_{1}^{2}+p_{22} r_{2}^{2}+s_{2} r_{1}^{4}\right)
\end{array}\right.
$$

where

$$
p_{11}=P_{11}, p_{12}=P_{12}, p_{21}=\Re\left(P_{21}\right), p_{22}=\Re\left(P_{22}\right), s_{1}=S_{1}, s_{2}=\Re\left(S_{2}\right) .
$$

The values of $p_{j k}$ and $s_{j}$, for $j, k=1,2$, and the quantities

$$
\theta=\frac{p_{12}}{p_{22}}, \delta=\frac{p_{21}}{p_{11}}, \Theta=\frac{s_{1}}{p_{22}^{2}}, \Delta=\frac{s_{2}}{p_{11}^{2}}
$$

indicate in which bifurcation scenario we are (see Section 8.6.2 in [7]).

In the "simple" case where $p_{11} p_{22}>0$, there are five topologically different bifurcation diagrams of the truncated amplitude system (10). Each case corresponds with a region in the $(\theta, \delta)$-plane, see Figure $2(\mathrm{a})$. The parametric portraits belonging to the different regions can be seen in Figure 3 (a), with corresponding phase portraits in the $\left(r_{1}, r_{2}\right)$-plane in Figure $3(\mathrm{~b})$. The phase portraits are only shown for the case when $p_{11}<0$ and $p_{22}<0$. The case $p_{11}>0$ and $p_{22}>0$ can be reduced to the considered one by reversing time.

In the "difficult" case where $p_{11} p_{22}<0$ however, there are six essentially different bifurcation diagrams. The regions in the $(\theta, \delta)$-plane are shown in Figure 2 (b). The related parametric portraits and phase portraits of (10) are given in Figure 4 . Only the case $p_{11}>0$ and $p_{22}<0$ is presented, to which the opposite one can be easily reduced.

We note that Section 8.6.2 in [7] for the "difficult" case contains a few errors in the figures and in the asymptotic expression for the heteroclinic bifurcation curve $^{3}$. Therefore, for completeness, we provide the figures here,

\footnotetext{
${ }^{3}$ Unfortunately, there is also a minor misprint in our earlier "correction" [8] for the heteroclinic curve given in [7].
} 
and derive the quadratic asymptotics of the Hopf $(C)$ and heteroclinic $(Y)$ bifurcation curves in Appendix A.

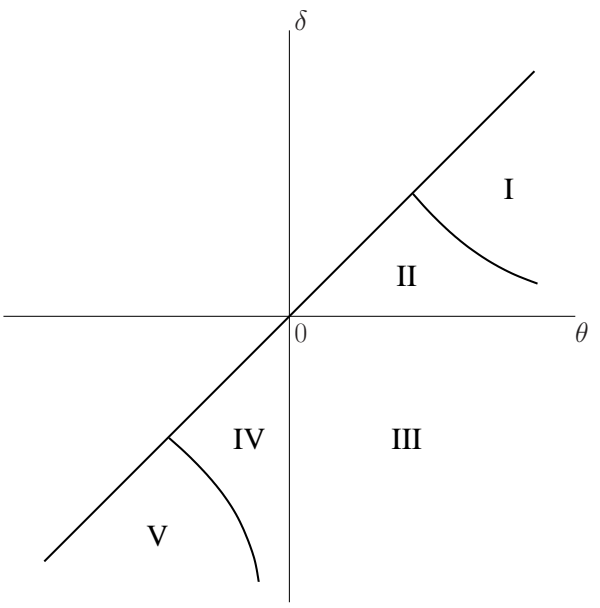

(a)

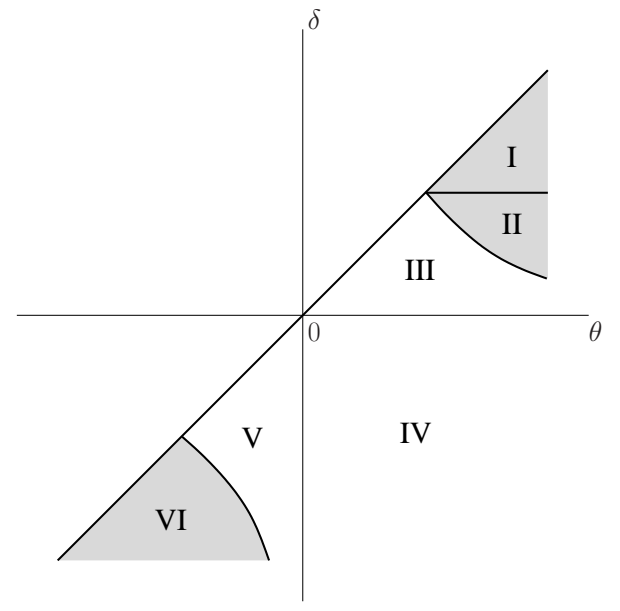

(b)

Figure 2: (a) the five subregions in the $(\theta, \delta)$-plane in the "simple" case; (b) the six subregions in the $(\theta, \delta)$-plane in the "difficult" case.

The critical values of $P_{j k}$ and $S_{j}$ can be expressed in terms of the coefficients of (5) as (see page 356 in [7])

$$
P_{11}=a_{300}, P_{12}=a_{111}, \Re\left(P_{21}\right)=\Re\left(b_{210}\right), \Re\left(P_{22}\right)=\Re\left(b_{021}\right),
$$

and

$$
\begin{aligned}
S_{1} & =a_{122}+a_{111}\left(\frac{\Re\left(b_{221}\right)}{\Re\left(b_{210}\right)}-2 \frac{\Re\left(b_{032}\right)}{\Re\left(b_{021}\right)}-\frac{a_{500} \Re\left(b_{021}\right)}{a_{300} \Re\left(b_{210}\right)}\right), \\
\Re\left(S_{2}\right) & =\Re\left(b_{410}\right)+\Re\left(b_{210}\right)\left(\frac{a_{311}}{a_{111}}-2 \frac{a_{500}}{a_{300}}-\frac{a_{300} \Re\left(b_{032}\right)}{a_{111} \Re\left(b_{021}\right)}\right) .
\end{aligned}
$$

The fifth-order terms in (5) determine the stability of the tori in the "difficult" cases. In fact, the sign of the first Lyapunov coefficient for the Neimark-Sacker bifurcation is given by

$$
\operatorname{sign} l_{1}=-\operatorname{sign}(\theta(\theta(\theta-1) \Delta+\delta(\delta-1) \Theta)) \text {. }
$$

The output of MATCONT is $\left(p_{11}, p_{22}, \theta, \delta \text {, sign } l_{1}\right)^{4}$.

\footnotetext{
${ }^{4}$ Remark that $\operatorname{sign} l_{1}=\mathrm{NaN}$ is reported when terms up to only third order are computed.
} 

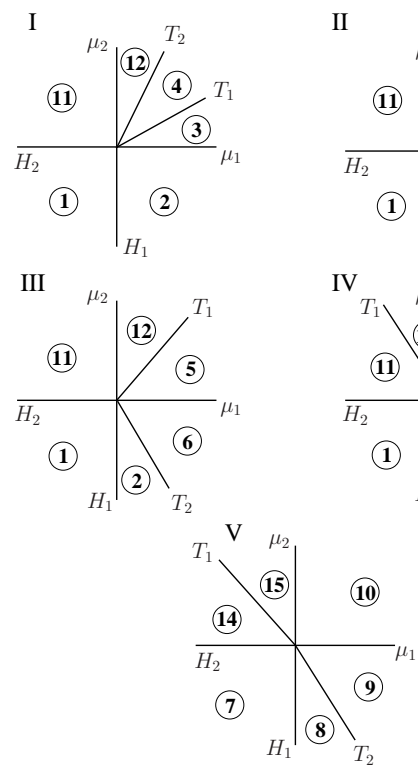

(a)
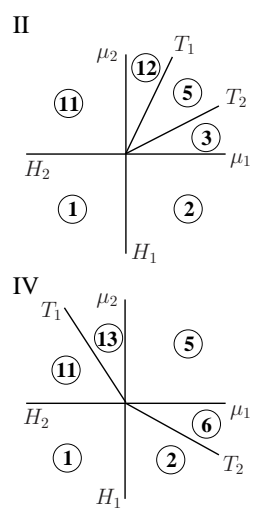

(10)

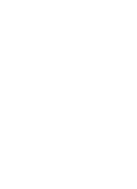

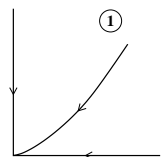
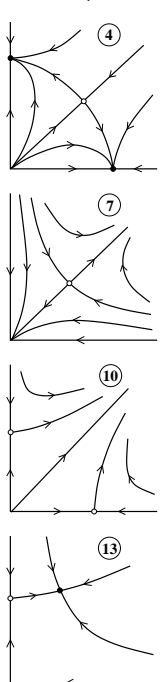

(1)

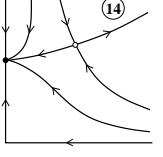

(b)

Figure 3: Bifurcation diagrams of the amplitude system (10) for the PDNS and NSNS bifurcations: (a) parametric portraits in the "simple" case; (b) phase portraits in the "simple" case.

For PDNS we have an interpretation analogous to LPNS, but the invariant sets may be "doubled". The origin always corresponds the original limit cycle. Other fixed points on the horizontal axis represent the period-doubled limit cycles, while a fixed point on the vertical axis corresponds to a $\mathbb{T}^{2}$. Fixed points off the coordinate axes correspond to doubled tori $\mathbb{T}^{2}$ and periodic orbits correspond to $\mathbb{T}^{3}$. As in the LPNS case, Figures 3 and 4 present bifurcations of the truncated amplitude system that only approximates the full normalized unfolding. In particular, one has to be carefull with 'torus doubling', which is in fact a complicated quasiperiodic bifurcation [20, 21].

\subsection{3. $N S N S$}

Generically, a two-parameter unfolding of (1) near this bifurcation restricted to the center manifold is smoothly orbitally equivalent to a system 

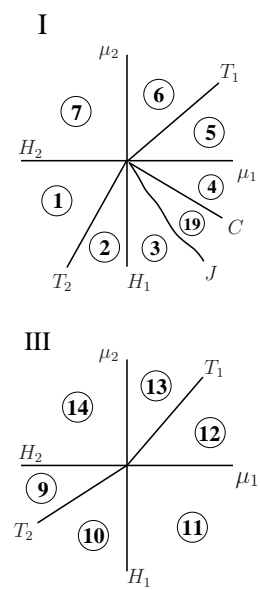

V

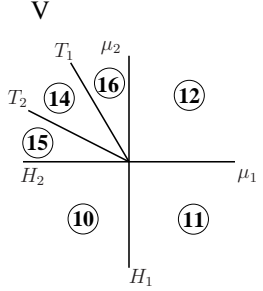

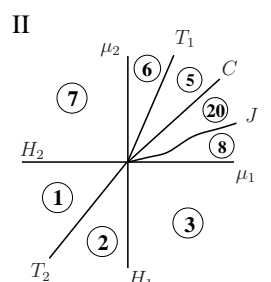
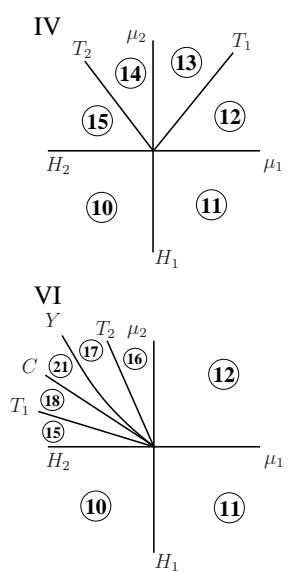

(a)
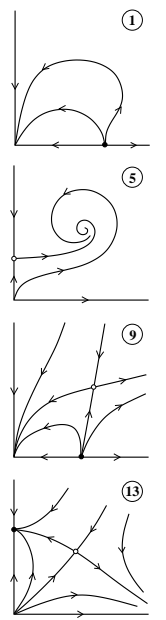

(17)

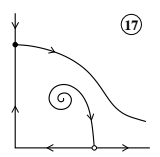

(b)

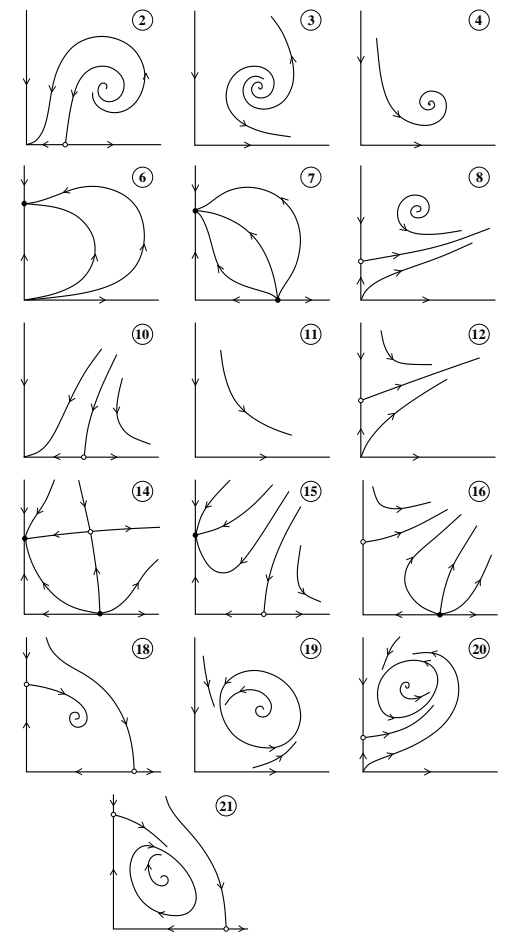

Figure 4: Bifurcation diagrams of the amplitude system (10) for the PDNS and NSNS bifurcations: (a) parametric portraits in the "difficult" case; (b) phase portraits in the "difficult" case. The quadratic asymptotics of the bifurcation curves $C$ and $Y$ are given in Appendix A.

in which the equations for the transverse coordinates have the form

$$
\left\{\begin{aligned}
\dot{v}_{1}= & \left(\mu_{1}+i \omega_{1}\right) v_{1}+P_{11} v_{1}\left|v_{1}\right|^{2}+P_{12} v_{1}\left|v_{2}\right|^{2}+i R_{1} v_{1}\left|v_{1}\right|^{4}+S_{1} v_{1}\left|v_{2}\right|^{4} \\
& +O\left(\|(v, \bar{v})\|^{6}\right), \\
\dot{v}_{2}= & \left(\mu_{2}+i \omega_{2}\right) v_{2}+P_{21} v_{2}\left|v_{1}\right|^{2}+P_{22} v_{2}\left|v_{2}\right|^{2}+S_{2} v_{2}\left|v_{1}\right|^{4}+i R_{2} v_{2}\left|v_{2}\right|^{4} \\
& +O\left(\|(v, \bar{v})\|^{6}\right),
\end{aligned}\right.
$$

where the $O$-terms are $T$-periodic in $\tau$. Neglecting this periodicity, system (13) is the normal form for the Hopf-Hopf bifurcation of equilibria (cf. Lemma 8.14 on page 354 in [7]). 
The truncated amplitude system for (13) is the same as (10), where now

$$
\begin{aligned}
& p_{11}=\Re\left(P_{11}\right)=\Re\left(a_{2100}\right), p_{12}=\Re\left(P_{12}\right)=\Re\left(a_{1011}\right), \\
& p_{21}=\Re\left(P_{21}\right)=\Re\left(b_{1110}\right), p_{22}=\Re\left(P_{22}\right)=\Re\left(b_{0021}\right),
\end{aligned}
$$

and

$$
\begin{aligned}
s_{1} & =\Re\left(S_{1}\right) \\
& =\Re\left(a_{1022}\right)+\Re\left(a_{1011}\right)\left(\frac{\Re\left(b_{1121}\right)}{\Re\left(b_{1110}\right)}-2 \frac{\Re\left(b_{0032}\right)}{\Re\left(b_{0021}\right)}-\frac{\Re\left(a_{3200}\right) \Re\left(b_{0021}\right)}{\Re\left(a_{2100}\right) \Re\left(b_{1110}\right)}\right), \\
s_{2} & =\Re\left(S_{2}\right) \\
& =\Re\left(b_{2210}\right)+\Re\left(b_{1110}\right)\left(\frac{\Re\left(a_{2111}\right)}{\Re\left(a_{1011}\right)}-2 \frac{\Re\left(a_{3200}\right)}{\Re\left(a_{2100}\right)}-\frac{\Re\left(a_{2100}\right) \Re\left(b_{0032}\right)}{\Re\left(a_{1011}\right) \Re\left(b_{0021}\right)}\right) .
\end{aligned}
$$

The output of MATCONT is $\left(p_{11}, p_{22}, \theta, \delta \text {, sign } l_{1}\right)^{5}$.

Although the phase portraits of the truncated amplitude system are the same as for PDNS, their interpretation is slightly different, since they 'live' in the $\left(\left|v_{1}\right|,\left|v_{2}\right|\right)$-plane. Here, on both axes the fixed points correspond to invariant $2 \mathrm{D}$ tori $\mathbb{T}^{2}$ for the original system. Fixed points off the coordinate axes and limit cycles correspond to $\mathbb{T}^{3}$ and $\mathbb{T}^{4}$, respectively. The usual remark on the approximate nature of the bifurcation diagrams applies here as well.

\section{Computation of critical coefficients}

As was mentioned in the previous section, the stability of the extra torus appearing in the "difficult" cases is determined by third order terms for the LPNS bifurcation and fifth order terms for the PDNS and NSNS bifurcations. In the "simple" cases, second order derivatives are sufficient to determine the behaviour in the LPNS bifurcations and third order derivatives are sufficient in the PDNS and NSNS bifurcations. Therefore, we restrict our computations in this section to second order terms in the LPNS case and up to and including third order terms in the PDNS and NSNS cases. The expressions of the third order coefficients for LPNS and fourth and fifth order coefficients for PDNS and NSNS are given in Appendix C in [18]. Remark that for efficiency reasons these higher order coefficients are not computed in MATCONT, unless explicitly requested by the user.

\footnotetext{
${ }^{5}$ Remark that $\operatorname{sign} l_{1}=\mathrm{NaN}$ is reported when terms up to only third order are computed.
} 


\section{1. $L P N S$}

The four-dimensional critical center manifold $W^{c}(\Gamma)$ at the LPNS bifurcation can be parametrized locally by $\left(\xi_{1}, \xi_{2}, \tau\right) \in \mathbb{R} \times \mathbb{C} \times[0, T]$ as

$$
u=u_{0}(\tau)+\xi_{1} v_{1}(\tau)+\xi_{2} v_{2}(\tau)+\bar{\xi}_{2} \bar{v}_{2}(\tau)+H\left(\xi_{1}, \xi_{2}, \tau\right),
$$

where $H$ satisfies $H\left(\xi_{1}, \xi_{2}, T\right)=H\left(\xi_{1}, \xi_{2}, 0\right)$ and has the Taylor expansion

$$
H\left(\xi_{1}, \xi_{2}, \tau\right)=\sum_{2 \leq i+j+k \leq 3} \frac{1}{i ! j ! k !} h_{i j k}(\tau) \xi_{1}^{i} \xi_{2}^{j} \bar{\xi}_{2}^{k}+O\left(\|\xi\|^{4}\right),
$$

where the eigenfunctions $v_{1}$ and $v_{2}$ are defined by

$$
\left\{\begin{aligned}
\dot{v}_{1}-A(\tau) v_{1}-F\left(u_{0}\right) & =0, \tau \in[0, T] \\
v_{1}(T)-v_{1}(0) & =0 \\
\int_{0}^{T}\left\langle v_{1}, F\left(u_{0}\right)\right\rangle d \tau & =0
\end{aligned}\right.
$$

and

$$
\left\{\begin{aligned}
\dot{v}_{2}-A(\tau) v_{2}+i \omega v_{2} & =0, \tau \in[0, T] \\
v_{2}(T)-v_{2}(0) & =0 \\
\int_{0}^{T}\left\langle v_{2}, v_{2}\right\rangle d \tau-1 & =0
\end{aligned}\right.
$$

In the codim 2 case considered here, the functions $v_{1}$ and $v_{2}$ exist because of Lemma 2 of [14]. The functions $h_{i j k}$ will be found by solving appropriate BVPs, assuming that (2) restricted to $W^{c}(\Gamma)$ has the normal form (4).

The coefficients of the normal form arise from the solvability conditions for the BVPs as integrals of scalar products over the interval $[0, T]$. Specifically, those scalar products involve among other things the quadratic and cubic terms of (3) near the periodic solution $u_{0}$, the generalized eigenfunction $v_{1}$ and eigenfunction $v_{2}$, and the adjoint eigenfunctions $\varphi^{*}, v_{1}^{*}$ and $v_{2}^{*}$ as solutions of the problems

$$
\left\{\begin{aligned}
\dot{\varphi}^{*}+A^{\mathrm{T}}(\tau) \varphi^{*} & =0, \tau \in[0, T] \\
\varphi^{*}(T)-\varphi^{*}(0) & =0 \\
\int_{0}^{T}\left\langle\varphi^{*}, v_{1}\right\rangle d \tau-1 & =0
\end{aligned}\right.
$$

and

$$
\left\{\begin{aligned}
\dot{v}_{1}^{*}+A^{\mathrm{T}}(\tau) v_{1}^{*}+\varphi^{*} & =0, \tau \in[0, T] \\
v_{1}^{*}(T)-v_{1}^{*}(0) & =0 \\
\int_{0}^{T}\left\langle v_{1}^{*}, v_{1}\right\rangle d \tau & =0
\end{aligned}\right.
$$


and

$$
\left\{\begin{aligned}
\dot{v}_{2}^{*}+A^{\mathrm{T}}(\tau) v_{2}^{*}+i \omega v_{2}^{*} & =0, \tau \in[0, T] \\
v_{2}^{*}(T)-v_{2}^{*}(0) & =0 \\
\int_{0}^{T}\left\langle v_{2}^{*}, v_{2}\right\rangle d \tau-1 & =0 .
\end{aligned}\right.
$$

In what follows we will make use of the orthogonality condition

$$
\int_{0}^{T}\left\langle\varphi^{*}, F\left(u_{0}\right)\right\rangle d \tau=0
$$

and the normalization condition

$$
\int_{0}^{T}\left\langle v_{1}^{*}, F\left(u_{0}\right)\right\rangle d \tau=1
$$

which can be easily obtained from (16), (18) and (19).

To derive the normal form coefficients we write down the homological equation and compare term by term. We therefore substitute (14) into (2), using (3), (4) and (15). By collecting the constant and linear terms we get the identities

$$
\dot{u}_{0}=F\left(u_{0}\right), \quad \dot{v}_{1}-F\left(u_{0}\right)=A(\tau) v_{1}, \quad \dot{v}_{2}+i \omega v_{2}=A(\tau) v_{2},
$$

and the complex conjugate of the last equation.

By collecting the $\xi_{1}^{2}$-terms we find an equation for $h_{200}$

$$
\dot{h}_{200}-A(\tau) h_{200}=B\left(\tau ; v_{1}, v_{1}\right)-2 a_{200} v_{1}-2 \alpha_{200} \dot{u}_{0}+2 \dot{v}_{1},
$$

to be solved in the space of functions satisfying $h_{200}(T)=h_{200}(0)$. In this space, the differential operator $\frac{d}{d \tau}-A(\tau)$ is singular and its null-space is spanned by $\dot{u}_{0}$. The Fredholm solvability condition

$$
\int_{0}^{T}\left\langle\varphi^{*}, B\left(\tau ; v_{1}, v_{1}\right)-2 a_{200} v_{1}-2 \alpha_{200} \dot{u}_{0}+2 \dot{v}_{1}\right\rangle d \tau=0
$$

allows one to calculate the coefficient $a_{200}$ in (4) due to the required normalization in (18), i.e.

$$
a_{200}=\frac{1}{2} \int_{0}^{T}\left\langle\varphi^{*}, B\left(\tau ; v_{1}, v_{1}\right)+2 A(\tau) v_{1}\right\rangle d \tau
$$


taking (16) and (21) into account. With $a_{200}$ defined in this way, let $h_{200}$ be a solution of (23) in the space of functions satisfying $h_{200}(0)=h_{200}(T)$. Notice that if $h_{200}$ is a solution of (23), then also $h_{200}+\varepsilon_{1} F\left(u_{0}\right)$ satisfies (23), since $F\left(u_{0}\right)$ is in the kernel of the operator $\frac{d}{d \tau}-A(\tau)$. In order to obtain a unique solution (without a component along the null eigenspace) we impose the following orthogonality condition which determines the value of $\varepsilon_{1}$

$$
\int_{0}^{T}\left\langle v_{1}^{*}, h_{200}\right\rangle d \tau=0,
$$

since (22) holds. Thus $h_{200}$ is the unique solution of the BVP

$$
\left\{\begin{aligned}
\dot{h}_{200}-A(\tau) h_{200}-B\left(\tau ; v_{1}, v_{1}\right)-2 A(\tau) v_{1} & \\
+2 a_{200} v_{1}+2 \alpha_{200} \dot{u}_{0}-2 \dot{u}_{0} & =0, \tau \in[0, T], \\
h_{200}(T)-h_{200}(0) & =0, \\
\int_{0}^{T}\left\langle v_{1}^{*}, h_{200}\right\rangle d \tau & =0 .
\end{aligned}\right.
$$

By collecting the $\xi_{2}^{2}$-terms (or $\bar{\xi}_{2}^{2}$-terms) we find an equation for $h_{020}$

$$
\dot{h}_{020}-A(\tau) h_{020}+2 i \omega h_{020}=B\left(\tau ; v_{2}, v_{2}\right),
$$

(or its complex conjugate). This equation has a unique solution $h_{020}$ satisfying $h_{020}(T)=h_{020}(0)$, since due to the spectral assumptions $e^{2 i \omega T}$ is not a multiplier of the critical cycle. Thus, $h_{020}$ can be found by solving

$$
\left\{\begin{aligned}
\dot{h}_{020}-A(\tau) h_{020}+2 i \omega h_{020}-B\left(\tau ; v_{2}, v_{2}\right) & =0, \tau \in[0, T], \\
h_{020}(T)-h_{020}(0) & =0 .
\end{aligned}\right.
$$

By collecting the $\xi_{1} \xi_{2}$-terms we obtain an equation for $h_{110}$

$$
\dot{h}_{110}-A(\tau) h_{110}+i \omega h_{110}=B\left(\tau ; v_{1}, v_{2}\right)-b_{110} v_{2}+\dot{v}_{2}+i \omega v_{2},
$$

to be solved in the space of functions satisfying $h_{110}(T)=h_{110}(0)$. In this space, the differential operator $\frac{d}{d \tau}-A(\tau)+i \omega$ is singular, since $e^{i \omega T}$ is a critical multiplier. So we can impose the following Fredholm solvability condition

$$
\int_{0}^{T}\left\langle v_{2}^{*}, B\left(\tau ; v_{1}, v_{2}\right)-b_{110} v_{2}+\dot{v}_{2}+i \omega v_{2}\right\rangle d \tau=0,
$$

which due to the normalization condition in (20) determines the value of the normal form coefficient $b_{110}$, yielding

$$
b_{110}=\int_{0}^{T}\left\langle v_{2}^{*}, B\left(\tau ; v_{1}, v_{2}\right)+A(\tau) v_{2}\right\rangle d \tau
$$


The nullspace belonging to the operator $\frac{d}{d \tau}-A(\tau)+i \omega$ is one-dimensional and spanned by $v_{2}$. To determine $h_{110}$ uniquely, we need to impose an orthogonality condition with a vector whose inproduct with $v_{2}$ is non-zero. The function $v_{2}^{*}$ can be choosen because of the normalisation condition in (20). In fact, $h_{110}$ only appears in the normal form coefficient $b_{210}$ (see Appendix C.1 in [18]), and a different normalization of $h_{110}$ does not influence the value of that normal form coefficient. Therefore, we define $h_{110}$ as the unique solution of the BVP

$$
\left\{\begin{aligned}
\dot{h}_{110}-A(\tau) h_{110}+i \omega h_{110}-B\left(\tau ; v_{1}, v_{2}\right)+b_{110} v_{2}-A(\tau) v_{2} & =0, \tau \in[0, T], \\
h_{110}(T)-h_{110}(0) & =0 \\
\int_{0}^{T}\left\langle v_{2}^{*}, h_{110}\right\rangle d \tau & =0 .
\end{aligned}\right.
$$

By collecting the $\left|\xi_{2}\right|^{2}$-terms we obtain a singular equation for $h_{011}$, namely

$$
\dot{h}_{011}-A(\tau) h_{011}=B\left(\tau ; v_{2}, \bar{v}_{2}\right)-a_{011} v_{1}-\alpha_{011} \dot{u}_{0},
$$

to be solved in the space of functions satisfying $h_{011}(T)=h_{011}(0)$. The nontrivial kernel of the operator $\frac{d}{d \tau}-A(\tau)$ is spanned by $\dot{u}_{0}$. So, the following Fredholm solvability condition is involved

$$
\int_{0}^{T}\left\langle\varphi^{*}, B\left(\tau ; v_{2}, \bar{v}_{2}\right)-a_{011} v_{1}-\alpha_{011} \dot{u}_{0}\right\rangle d \tau=0
$$

which gives us the expression for the normal form coefficient $a_{011}$, i.e.

$$
a_{011}=\int_{0}^{T}\left\langle\varphi^{*}, B\left(\tau ; v_{2}, \bar{v}_{2}\right)\right\rangle d \tau
$$

We impose the orthogonality condition with the adjoint generalized eigenfunction $v_{1}^{*}$ to obtain $h_{011}$ as the unique solution of

$$
\left\{\begin{aligned}
\dot{h}_{011}-A(\tau) h_{011}-B\left(\tau ; v_{2}, \bar{v}_{2}\right)+a_{011} v_{1}+\alpha_{011} \dot{u}_{0} & =0, \tau \in[0, T] \\
h_{011}(T)-h_{011}(0) & =0 \\
\int_{0}^{T}\left\langle v_{1}^{*}, h_{011}\right\rangle d \tau & =0
\end{aligned}\right.
$$

We remark that the values of $\alpha_{200}$ and $\alpha_{011}$ are not determined by the homological equation. We therefore put them equal to zero.

Third order coefficients are only needed to determine the stability of the torus, if this torus exists. They can be found in Appendix $\mathrm{C}$ in [18]. 


\section{2. $P D N S$}

The four-dimensional critical center manifold $W^{c}(\Gamma)$ at the PDNS bifurcation can be parametrized locally by $\left(\xi_{1}, \xi_{2}, \tau\right) \in \mathbb{R} \times \mathbb{C} \times[0,2 T]$ as

$$
u=u_{0}(\tau)+\xi_{1} v_{1}(\tau)+\xi_{2} v_{2}(\tau)+\bar{\xi}_{2} \bar{v}_{2}(\tau)+H\left(\xi_{1}, \xi_{2}, \tau\right),
$$

where $H$ satisfies $H\left(\xi_{1}, \xi_{2}, 2 T\right)=H\left(\xi_{1}, \xi_{2}, 0\right)$ and has the Taylor expansion

$$
H\left(\xi_{1}, \xi_{2}, \tau\right)=\sum_{2 \leq i+j+k \leq 5} \frac{1}{i ! j ! k !} h_{i j k}(\tau) \xi_{1}^{i} \xi_{2}^{j} \bar{\xi}_{2}^{k}+O\left(\|\xi\|^{6}\right),
$$

while the eigenfunctions $v_{1}$ and $v_{2}$ are defined by

$$
\left\{\begin{aligned}
\dot{v}_{1}-A(\tau) v_{1} & =0, \tau \in[0, T] \\
v_{1}(T)+v_{1}(0) & =0 \\
\int_{0}^{T}\left\langle v_{1}, v_{1}\right\rangle d \tau-1 & =0
\end{aligned}\right.
$$

with $v_{1}(\tau+T)=-v_{1}(\tau)$ for $\tau \in[0, T]$ and (17).

The functions $v_{1}$ and $v_{2}$ exist because of Lemma 5 of [14]. The functions $h_{i j k}$ can be found by solving appropriate BVPs, assuming that (2) restricted to $W^{c}(\Gamma)$ has the normal form (5). Moreover, $u\left(\tau, \xi_{1}, \xi_{2}, \bar{\xi}_{2}\right)=$ $u\left(\tau+T,-\xi_{1}, \xi_{2}, \bar{\xi}_{2}\right)$ so that

$$
h_{i j k}(\tau)=(-1)^{i} h_{i j k}(\tau+T),
$$

for $\tau \in[0, T]$. Therefore, we can restrict our computations to the interval $[0, T]$ instead of $[0,2 T]$.

The coefficients of the normal form arise from the solvability conditions for the BVPs as integrals of scalar products over the interval $[0, T]$. Specifically, those scalar products involve among other things the quadratic and cubic terms of (3) near the periodic solution $u_{0}, v_{1}, v_{2}$, and the adjoint eigenfunctions $\varphi^{*}, v_{1}^{*}$ and $v_{2}^{*}$ as solutions of the problems

$$
\left\{\begin{aligned}
\dot{\varphi}^{*}+A^{\mathrm{T}}(\tau) \varphi^{*} & =0, \tau \in[0, T], \\
\varphi^{*}(T)-\varphi^{*}(0) & =0 \\
\int_{0}^{T}\left\langle\varphi^{*}, F\left(u_{0}\right)\right\rangle d \tau-1 & =0
\end{aligned}\right.
$$

and

$$
\left\{\begin{aligned}
\dot{v}_{1}^{*}+A^{\mathrm{T}}(\tau) v_{1}^{*} & =0, \tau \in[0, T] \\
v_{1}^{*}(T)+v_{1}^{*}(0) & =0 \\
\int_{0}^{T}\left\langle v_{1}^{*}, v_{1}\right\rangle d \tau-1 & =0
\end{aligned}\right.
$$


and (20).

By collecting the constant and linear terms we get the identities

$$
\dot{u}_{0}=F\left(u_{0}\right), \quad \dot{v}_{1}=A(\tau) v_{1}, \quad \dot{v}_{2}+i \omega v_{2}=A(\tau) v_{2},
$$

and the complex conjugate of the last equation, which merely reflect the definition of $u_{0}$, (33) and (17).

By collecting the $\xi_{1}^{2}$-terms we obtain $h_{200}$ as the unique solution of the BVP

$$
\left\{\begin{aligned}
\dot{h}_{200}-A(\tau) h_{200}-B\left(\tau ; v_{1}, v_{1}\right)+2 \alpha_{200} \dot{u}_{0} & =0, \tau \in[0, T], \\
h_{200}(T)-h_{200}(0) & =0 \\
\int_{0}^{T}\left\langle\varphi^{*}, h_{200}\right\rangle d \tau & =0,
\end{aligned}\right.
$$

where

$$
\alpha_{200}=\frac{1}{2} \int_{0}^{T}\left\langle\varphi^{*}, B\left(\tau ; v_{1}, v_{1}\right)\right\rangle d \tau .
$$

By collecting the $\xi_{2}^{2}$-terms (or $\bar{\xi}_{2}^{2}$-terms) we find $h_{020}$ as solution of

$$
\left\{\begin{aligned}
\dot{h}_{020}-A(\tau) h_{020}+2 i \omega h_{020}-B\left(\tau ; v_{2}, v_{2}\right) & =0, \tau \in[0, T], \\
h_{020}(T)-h_{020}(0) & =0 .
\end{aligned}\right.
$$

The BVP found by comparing the $\xi_{1} \xi_{2}$-terms is given by

$$
\left\{\begin{aligned}
\dot{h}_{110}-A(\tau) h_{110}+i \omega h_{110}-B\left(\tau ; v_{1}, v_{2}\right) & =0, \tau \in[0, T], \\
h_{110}(T)+h_{110}(0) & =0,
\end{aligned}\right.
$$

The $\left|\xi_{2}\right|^{2}$-terms lead to a singular equation for $h_{011}$ such that the expression for the normal form coefficient $\alpha_{011}$ can be derived as

$$
\alpha_{011}=\int_{0}^{T}\left\langle\varphi^{*}, B\left(\tau ; v_{2}, \bar{v}_{2}\right)\right\rangle d \tau .
$$

Funtion $h_{011}$ is then the unique solution of

$$
\left\{\begin{aligned}
\dot{h}_{011}-A(\tau) h_{011}-B\left(\tau ; v_{2}, \bar{v}_{2}\right)+\alpha_{011} \dot{u}_{0} & =0, \tau \in[0, T], \\
h_{011}(T)-h_{011}(0) & =0 \\
\int_{0}^{T}\left\langle\varphi^{*}, h_{011}\right\rangle d \tau & =0 .
\end{aligned}\right.
$$

We have now examined all order two terms, and continue with the order three terms. 
Collecting the $\xi_{1}^{3}$-terms gives an equation for $h_{300}$ and allows us to obtain the following formula for the normal form coefficient $a_{300}$ in (5):

$$
a_{300}=\frac{1}{6} \int_{0}^{T}\left\langle v_{1}^{*}, C\left(\tau ; v_{1}, v_{1}, v_{1}\right)+3 B\left(\tau ; v_{1}, h_{200}\right)-6 \alpha_{200} A(\tau) v_{1}\right\rangle d \tau .
$$

$h_{300}$ is then found as the unique solution of

$$
\left\{\begin{aligned}
\dot{h}_{300}-A(\tau) h_{300}-C\left(\tau ; v_{1}, v_{1}, v_{1}\right) & \\
-3 B\left(\tau ; v_{1}, h_{200}\right)+6 \alpha_{200} A(\tau) v_{1}+6 a_{300} v_{1} & =0, \tau \in[0, T] \\
h_{300}(T)+h_{300}(0) & =0 \\
\int_{0}^{T}\left\langle v_{1}^{*}, h_{300}\right\rangle d \tau & =0
\end{aligned}\right.
$$

The $\xi_{2}^{3}$ (or $\bar{\xi}_{2}^{3}$ )-terms from the homological equation give a BVP for $h_{030}$ :

$$
\left\{\begin{aligned}
\dot{h}_{030}-A(\tau) h_{030}+3 i \omega h_{030} & \\
-C\left(\tau ; v_{2}, v_{2}, v_{2}\right)-3 B\left(\tau ; v_{2}, h_{020}\right) & =0, \tau \in[0, T], \\
h_{030}(T)-h_{030}(0) & =0 .
\end{aligned}\right.
$$

By collecting the $\xi_{1}^{2} \xi_{2}$-terms we can derive the expression for the normal form coefficient $b_{210}$, namely

$$
\begin{aligned}
b_{210}= & \frac{1}{2} \int_{0}^{T}\left\langle v_{2}^{*}, C\left(\tau ; v_{1}, v_{1}, v_{2}\right)+B\left(\tau ; v_{2}, h_{200}\right)+2 B\left(\tau ; v_{1}, h_{110}\right)\right. \\
& \left.-2 \alpha_{200} A(\tau) v_{2}\right\rangle d \tau
\end{aligned}
$$

We obtain $h_{210}$ as the unique solution of

$$
\left\{\begin{aligned}
\dot{h}_{210}-A(\tau) h_{210}+i \omega h_{210}-C\left(\tau ; v_{1}, v_{1}, v_{2}\right) & \\
-B\left(\tau ; v_{2}, h_{200}\right)-2 B\left(\tau ; v_{1}, h_{110}\right)+2 \alpha_{200} A(\tau) v_{2}+2 b_{210} v_{2} & =0, \tau \in[0, T], \\
h_{210}(T)-h_{210}(0) & =0 \\
\int_{0}^{T}\left\langle v_{2}^{*}, h_{210}\right\rangle d \tau & =0 .
\end{aligned}\right.
$$

Since $\xi_{1} \xi_{2}^{2}$ is not a term in the normal form (5), we will find a non-singular equation for $h_{120}$ when collecting the $\xi_{1} \xi_{2}^{2}$-terms from the homological equation, i.e.

$$
\left\{\begin{aligned}
\dot{h}_{120}-A(\tau) h_{120}+2 i \omega h_{120}-C\left(\tau ; v_{1}, v_{2}, v_{2}\right) & \\
-B\left(\tau ; v_{1}, h_{020}\right)-2 B\left(\tau ; v_{2}, h_{110}\right) & =0, \tau \in[0, T], \\
h_{120}(T)+h_{120}(0) & =0 .
\end{aligned}\right.
$$


The two remaining third order terms are the $\xi_{2}\left|\xi_{2}\right|^{2}$-terms and the $\xi_{1}\left|\xi_{2}\right|^{2}$ terms lead to the computation of the two remaining unknown third order normal form coefficients of (5), i.e.

$$
\begin{aligned}
b_{021}= & \frac{1}{2} \int_{0}^{T}\left\langle v_{2}^{*}, C\left(\tau ; v_{2}, v_{2}, \bar{v}_{2}\right)+B\left(\tau ; \bar{v}_{2}, h_{020}\right)+2 B\left(\tau ; v_{2}, h_{011}\right)\right. \\
& \left.-2 \alpha_{011} A(\tau) v_{2}\right\rangle d \tau
\end{aligned}
$$

and

$$
\begin{aligned}
a_{111}= & \int_{0}^{T}\left\langle v_{1}^{*}, C\left(\tau ; v_{1}, v_{2}, \bar{v}_{2}\right)+B\left(\tau ; v_{1}, h_{011}\right)+2 \Re\left(B\left(\tau ; v_{2}, h_{101}\right)\right)\right. \\
& \left.-\alpha_{011} A(\tau) v_{1}\right\rangle d \tau .
\end{aligned}
$$

Since we need both $h_{021}$ and $h_{111}$ for the computation of higher order normal form coefficients, we also write down their BVPs

$$
\left\{\begin{aligned}
\dot{h}_{021}-A(\tau) h_{021}+i \omega h_{021}-C\left(\tau ; v_{2}, v_{2}, \bar{v}_{2}\right) & \\
-B\left(\tau ; \bar{v}_{2}, h_{020}\right)-2 B\left(\tau ; v_{2}, h_{011}\right)+2 \alpha_{011} A(\tau) v_{2}+2 b_{021} v_{2} & =0, \tau \in[0, T], \\
h_{021}(T)-h_{021}(0) & =0 \\
\int_{0}^{T}\left\langle v_{2}^{*}, h_{021}\right\rangle d \tau & =0
\end{aligned}\right.
$$

and

$$
\left\{\begin{aligned}
\dot{h}_{111}-A(\tau) h_{111}-C\left(\tau ; v_{1}, v_{2}, \bar{v}_{2}\right)-B\left(\tau ; v_{1}, h_{011}\right) & \\
-2 \Re\left(B\left(\tau ; v_{2}, h_{101}\right)\right)+\alpha_{011} A(\tau) v_{1}+a_{111} v_{1} & =0, \tau \in[0, T], \\
h_{111}(T)+h_{111}(0) & =0 \\
\int_{0}^{T}\left\langle v_{1}^{*}, h_{111}\right\rangle d \tau & =0 .
\end{aligned}\right.
$$

The stability of a possibly existing torus depends on the fourth and fifth order coefficients, which are listed in Appendix C in [18].

\subsection{NSNS}

The five-dimensional critical center manifold $W^{c}(\Gamma)$ at the NSNS bifurcation can be parametrized locally by $(\xi, \tau) \in \mathbb{C}^{2} \times[0, T]$ as

$$
u=u_{0}(\tau)+\xi_{1} v_{1}(\tau)+\bar{\xi}_{1} \bar{v}_{1}(\tau)+\xi_{2} v_{2}(\tau)+\bar{\xi}_{2} \bar{v}_{2}(\tau)+H(\xi, \tau)
$$


where $H$ satisfies $H(\xi, T)=H(\xi, 0)$ and has the Taylor expansion

$$
H(\xi, \tau)=\sum_{2 \leq i+j+k+l \leq 5} \frac{1}{i ! j ! k ! l !} h_{i j k l}(\tau) \xi_{1}^{i} \bar{\xi}_{1}^{j} \xi_{2}^{k} \bar{\xi}_{2}^{l}+O\left(\|\xi\|^{6}\right),
$$

where the complex eigenfunctions $v_{1}$ and $v_{2}$ are given by

$$
\left\{\begin{aligned}
\dot{v}_{1}-A(\tau) v_{1}+i \omega_{1} v_{1} & =0, \tau \in[0, T] \\
v_{1}(T)-v_{1}(0) & =0 \\
\int_{0}^{T}\left\langle v_{1}, v_{1}\right\rangle d \tau-1 & =0
\end{aligned}\right.
$$

and (17).

The functions $v_{1}$ and $v_{2}$ exist because of Lemma 2 of [14]. The functions $h_{i j k l}$ will be found by solving appropriate BVPs, assuming that (2) restricted to $W^{c}(\Gamma)$ has the normal form (6).

The coefficients of the normal form arise from the solvability conditions for the BVPs as integrals of scalar products over the interval $[0, T]$. Specifically, those scalar products involve among other things the quadratic and cubic terms of (3) near the periodic solution $u_{0}$, the eigenfunctions $v_{1}$ and $v_{2}$, and the adjoint eigenfunctions $\varphi^{*}, v_{1}^{*}$ and $v_{2}^{*}$ as solution of the problems (35),

$$
\left\{\begin{aligned}
\dot{v}_{1}^{*}+A^{\mathrm{T}}(\tau) v_{1}^{*}+i \omega_{1} v_{1}^{*} & =0, \tau \in[0, T] \\
v_{1}^{*}(T)-v_{1}^{*}(0) & =0 \\
\int_{0}^{T}\left\langle v_{1}^{*}, v_{1}\right\rangle d \tau-1 & =0
\end{aligned}\right.
$$

and (20).

By collecting the constant and linear terms we get the identities

$$
\dot{u}_{0}=F\left(u_{0}\right), \quad \dot{v}_{1}+i \omega_{1} v_{1}=A(\tau) v_{1}, \quad \dot{v}_{2}+i \omega_{2} v_{2}=A(\tau) v_{2},
$$

and the complex conjugates of the last two equations. The above equations merely reflect the definition of $u_{0}$ and the first equations in (55), (17).

By collecting the $\xi_{1}^{2}$ (or $\bar{\xi}_{1}^{2}$-terms)-terms we find a BVP for $h_{2000}$ :

$$
\left\{\begin{aligned}
\dot{h}_{2000}-A(\tau) h_{2000}+2 i \omega_{1} h_{2000}-B\left(\tau ; v_{1}, v_{1}\right) & =0, \tau \in[0, T], \\
h_{2000}(T)-h_{2000}(0) & =0 .
\end{aligned}\right.
$$

The function $h_{0200}$ is just the complex conjugate of the function $h_{2000}$. Analogously, by comparing the $\xi_{2}^{2}$-terms, we find that $h_{0020}$ is the unique solution of

$$
\left\{\begin{aligned}
\dot{h}_{0020}-A(\tau) h_{0020}+2 i \omega_{2} h_{0020}-B\left(\tau ; v_{2}, v_{2}\right) & =0, \tau \in[0, T], \\
h_{0020}(T)-h_{0020}(0) & =0 .
\end{aligned}\right.
$$


By collecting the $\left|\xi_{1}\right|^{2}$-terms we obtain a singular equation, as expected since this term is present in the normal form (6). From the Fredholm solvability condition follows that we can calculate parameter $\alpha_{1100}$ as

$$
\alpha_{1100}=\int_{0}^{T}\left\langle\varphi^{*}, B\left(\tau ; v_{1}, \bar{v}_{1}\right)\right\rangle d \tau
$$

With this value of $\alpha_{1100}$ we obtain $h_{1100}$ as the unique solution of the BVP

$$
\left\{\begin{aligned}
\dot{h}_{1100}-A(\tau) h_{1100}-B\left(\tau ; v_{1}, \bar{v}_{1}\right)+\alpha_{1100} \dot{u}_{0} & =0, \tau \in[0, T] \\
h_{1100}(T)-h_{1100}(0) & =0 \\
\int_{0}^{T}\left\langle\varphi^{*}, h_{1100}\right\rangle d \tau & =0 .
\end{aligned}\right.
$$

Analogously, function $h_{0011}$ can be obtained by solving

$$
\left\{\begin{aligned}
\dot{h}_{0011}-A(\tau) h_{0011}-B\left(\tau ; v_{2}, \bar{v}_{2}\right)+\alpha_{0011} \dot{u}_{0} & =0, \tau \in[0, T] \\
h_{0011}(T)-h_{0011}(0) & =0 \\
\int_{0}^{T}\left\langle\varphi^{*}, h_{0011}\right\rangle d \tau & =0
\end{aligned}\right.
$$

with

$$
\alpha_{0011}=\int_{0}^{T}\left\langle\varphi^{*}, B\left(\tau ; v_{2}, \bar{v}_{2}\right)\right\rangle d \tau \text {. }
$$

From collecting the $\xi_{1} \xi_{2}$-terms follows that $h_{1010}$ can be found by solving

$$
\left\{\begin{aligned}
\dot{h}_{1010}-A(\tau) h_{1010}+i \omega_{1} h_{1010}+i \omega_{2} h_{1010}-B\left(\tau ; v_{1}, v_{2}\right) & =0, \tau \in[0, T], \\
h_{1010}(T)-h_{1010}(0) & =0 .
\end{aligned}\right.
$$

We note that $h_{0101}=\overline{h_{1010}}$.

The last second order term is found from the $\xi_{1} \bar{\xi}_{2}$-terms and is a nonsingular differential equation, such that

$$
\left\{\begin{aligned}
\dot{h}_{1001}-A(\tau) h_{1001}+i \omega_{1} h_{1001}-i \omega_{2} h_{1001}-B\left(\tau ; v_{1}, \bar{v}_{2}\right) & =0, \tau \in[0, T], \\
h_{1001}(T)-h_{1001}(0) & =0 .
\end{aligned}\right.
$$

We now come to the third order terms. From the $\xi_{1}^{3}$ and $\xi_{2}^{3}$-terms we immediately get the BVPs for $h_{3000}$ and $h_{0030}$, namely

$$
\left\{\begin{aligned}
\dot{h}_{3000}-A(\tau) h_{3000}+3 i \omega_{1} h_{3000} & \\
-C\left(\tau ; v_{1}, v_{1}, v_{1}\right)-3 B\left(\tau ; v_{1}, h_{2000}\right) & =0, \tau \in[0, T] \\
h_{3000}(T)-h_{3000}(0) & =0
\end{aligned}\right.
$$


and

$$
\left\{\begin{aligned}
\dot{h}_{0030}-A(\tau) h_{0030}+3 i \omega_{2} h_{0030} & \\
-C\left(\tau ; v_{2}, v_{2}, v_{2}\right)-3 B\left(\tau ; v_{2}, h_{0020}\right) & =0, \tau \in[0, T], \\
h_{0030}(T)-h_{0030}(0) & =0 .
\end{aligned}\right.
$$

Since the $\xi_{1}\left|\xi_{1}\right|^{2}$-term is present in the normal form for the double NeimarkSacker bifurcation, a Fredholm solvability condition is involved, which determines $a_{2100}$ as

$$
\begin{aligned}
a_{2100}= & \frac{1}{2} \int_{0}^{T}\left\langle v_{1}^{*}, C\left(\tau ; v_{1}, v_{1}, \bar{v}_{1}\right)+2 B\left(\tau ; v_{1}, h_{1100}\right)+B\left(\tau ; \bar{v}_{1}, h_{2000}\right)\right. \\
& \left.-2 \alpha_{1100} A(\tau) v_{1}\right\rangle d \tau
\end{aligned}
$$

Therefore, we can compute $h_{2100}$ as the unique solution of the BVP

$$
\left\{\begin{aligned}
\dot{h}_{2100}-A(\tau) h_{2100}+i \omega_{1} h_{2100}-C\left(\tau ; v_{1}, v_{1}, \bar{v}_{1}\right) & \\
-2 B\left(\tau ; v_{1}, h_{1100}\right)-B\left(\tau ; \bar{v}_{1}, h_{2000}\right) & \\
+2 a_{2100} v_{1}+2 \alpha_{1100} A(\tau) v_{1} & =0, \tau \in[0, T], \\
h_{2100}(T)-h_{2100}(0) & =0, \\
\int_{0}^{T}\left\langle v_{1}^{*}, h_{2100}\right\rangle d \tau & =0 .
\end{aligned}\right.
$$

We can now immediately list the following four BVPs

$$
\begin{aligned}
& \left\{\begin{aligned}
\dot{h}_{2010}-A(\tau) h_{2010}+2 i \omega_{1} h_{2010}+i \omega_{2} h_{2010} & \\
-C\left(\tau ; v_{1}, v_{1}, v_{2}\right)-B\left(\tau ; v_{2}, h_{2000}\right)-2 B\left(\tau ; v_{1}, h_{1010}\right) & =0, \tau \in[0, T], \\
h_{2010}(T)-h_{2010}(0) & =0,
\end{aligned}\right. \\
& \left\{\begin{aligned}
\dot{h}_{2001}-A(\tau) h_{2001}+2 i \omega_{1} h_{2001}-i \omega_{2} h_{2001} & \\
-C\left(\tau ; v_{1}, v_{1}, \bar{v}_{2}\right)-B\left(\tau ; \bar{v}_{2}, h_{2000}\right)-2 B\left(\tau ; v_{1}, h_{1001}\right) & =0, \tau \in[0, T], \\
h_{2001}(T)-h_{2001}(0) & =0,
\end{aligned}\right. \\
& \left\{\begin{aligned}
\dot{h}_{1020}-A(\tau) h_{1020}+i \omega_{1} h_{1020}+2 i \omega_{2} h_{1020} & \\
-C\left(\tau ; v_{1}, v_{2}, v_{2}\right)-B\left(\tau ; v_{1}, h_{0020}\right)-2 B\left(\tau ; v_{2}, h_{1010}\right) & =0, \tau \in[0, T], \\
h_{1020}(T)-h_{1020}(0) & =0
\end{aligned}\right.
\end{aligned}
$$

and

$$
\left\{\begin{aligned}
\dot{h}_{0120}-A(\tau) h_{0120}-i \omega_{1} h_{0120}+2 i \omega_{2} h_{0120} & \\
-C\left(\tau ; \bar{v}_{1}, v_{2}, v_{2}\right)-B\left(\tau ; \bar{v}_{1}, h_{0020}\right)-2 B\left(\tau ; v_{2}, h_{0110}\right) & =0, \tau \in[0, T], \\
h_{0120}(T)-h_{0120}(0) & =0 .
\end{aligned}\right.
$$


The $\xi_{2}\left|\xi_{2}\right|^{2}$-terms from the homological equation make it possible to compute $b_{0021}$ as

$$
\begin{aligned}
b_{0021}= & \frac{1}{2} \int_{0}^{T}\left\langle v_{2}^{*}, C\left(\tau ; v_{2}, v_{2}, \bar{v}_{2}\right)+B\left(\tau ; \bar{v}_{2}, h_{0020}\right)+2 B\left(\tau ; v_{2}, h_{0011}\right)\right. \\
& \left.-2 \alpha_{0011} A(\tau) v_{2}\right\rangle d \tau
\end{aligned}
$$

with $h_{0021}$ as the unique solution of the BVP

$$
\left\{\begin{aligned}
\dot{h}_{0021}-A(\tau) h_{0021}+i \omega_{2} h_{0021}-C\left(\tau ; v_{2}, v_{2}, \bar{v}_{2}\right) & \\
-B\left(\tau ; \bar{v}_{2}, h_{0020}\right)-2 B\left(\tau ; v_{2}, h_{0011}\right) & \\
+2 b_{0021} v_{2}+2 \alpha_{0011} A(\tau) v_{2} & =0, \tau \in[0, T], \\
h_{0021}(T)-h_{0021}(0) & =0, \\
\int_{0}^{T}\left\langle v_{2}^{*}, h_{0021}\right\rangle d \tau & =0 .
\end{aligned}\right.
$$

The last two third order terms which we have to examine give us both the formula for a normal form coefficient. The first one, obtained from the $\left|\xi_{1}\right|^{2} \xi_{2}$-terms, gives us the BVP

$$
\left\{\begin{aligned}
\dot{h}_{1110}-A(\tau) h_{1110}+i \omega_{2} h_{1110}-C\left(\tau ; v_{1}, \bar{v}_{1}, v_{2}\right) & \\
-B\left(\tau ; v_{1}, h_{0110}\right)-B\left(\tau ; \bar{v}_{1}, h_{1010}\right)-B\left(\tau ; v_{2}, h_{1100}\right) & \\
+b_{1110} v_{2}+\alpha_{1100} A(\tau) v_{2} & =0, \tau \in[0, T], \\
h_{1110}(T)-h_{1110}(0) & =0 \\
\int_{0}^{T}\left\langle v_{2}^{*}, h_{1110}\right\rangle d \tau & =0
\end{aligned}\right.
$$

where from the solvability condition it follows that

$$
\begin{aligned}
b_{1110}= & \int_{0}^{T}\left\langle v_{2}^{*}, C\left(\tau ; v_{1}, \bar{v}_{1}, v_{2}\right)+B\left(\tau ; v_{1}, h_{0110}\right)+B\left(\tau ; \bar{v}_{1}, h_{1010}\right)\right. \\
& \left.+B\left(\tau ; v_{2}, h_{1100}\right)-\alpha_{1100} A(\tau) v_{2}\right\rangle d \tau
\end{aligned}
$$

Analogously, we obtain the BVP

$$
\left\{\begin{aligned}
\dot{h}_{1011}-A(\tau) h_{1011}+i \omega_{1} h_{1011}-C\left(\tau ; v_{1}, v_{2}, \bar{v}_{2}\right) & \\
-B\left(\tau ; v_{1}, h_{0011}\right)-B\left(\tau ; v_{2}, h_{1001}\right)-B\left(\tau ; \bar{v}_{2}, h_{1010}\right) & \\
+a_{1011} v_{1}+\alpha_{0011} A(\tau) v_{1} & =0, \tau \in[0, T], \\
h_{1011}(T)-h_{1011}(0) & =0 \\
\int_{0}^{T}\left\langle v_{1}^{*}, h_{1011}\right\rangle d \tau & =0
\end{aligned}\right.
$$


with

$$
\begin{aligned}
a_{1011}= & \int_{0}^{T}\left\langle v_{1}^{*}, C\left(\tau ; v_{1}, v_{2}, \bar{v}_{2}\right)+B\left(\tau ; v_{1}, h_{0011}\right)+B\left(\tau ; v_{2}, h_{1001}\right)\right. \\
& \left.+B\left(\tau ; \bar{v}_{2}, h_{1010}\right)-\alpha_{0011} A(\tau) v_{1}\right\rangle d \tau
\end{aligned}
$$

As before, the higher order coefficients which determine the stability of the torus can be found in Appendix $\mathrm{C}$ in [18].

\subsection{Implementation}

Numerical implementation of the formulas derived in the previous section requires the evaluation of integrals of scalar functions over $[0, T]$ and the solution of nonsingular linear BVPs with integral constraints. Such tasks can be carried out within the standard continuation software such as AUTO [1], CONTENT [22], and MATCONT [2]. In these software packages, periodic solutions to (1) are computed with the method of orthogonal collocation with piecewise polynomials applied to properly formulated BVPs [23, 24].

We have implemented our algorithms in MATCONT analogously to the eight cases with $n_{c} \leq 3$. For further details we refer to [17] where this is extensively discussed.

\section{Examples}

\subsection{Laser model}

In [25] a single-mode inversionless laser with a three-level phaser was studied and shown to operate in various modes. These modes are "off" (nonlasing), continuous waves, periodic, quasi-periodic and chaotic lasing. The model is a 9-dimensional system given by 3 real and 3 complex equations:

$$
\left\{\begin{array}{l}
\dot{\Omega}_{l}=-\frac{\gamma_{c a v}}{2} \Omega_{l}-g \Im\left(\sigma_{a b}\right) \\
\dot{\rho}_{a a}=R_{a}-\frac{i}{2}\left(\Omega_{l}\left(\sigma_{a b}-\sigma_{a b}^{*}\right)+\Omega_{p}\left(\sigma_{a c}-\sigma_{a c}^{*}\right)\right) \\
\dot{\rho}_{b b}=R_{b}+\frac{i}{2} \Omega_{l}\left(\sigma_{a b}-\sigma_{a b}^{*}\right) \\
\dot{\sigma}_{a b}=-\left(\gamma_{1}+i \Delta_{l}\right) \sigma_{a b}-\frac{i}{2}\left(\Omega_{l}\left(\rho_{a a}-\rho_{b b}\right)-\Omega_{p} \sigma_{c b}\right) \\
\dot{\sigma}_{a c}=-\left(\gamma_{2}+i \Delta_{p}\right) \sigma_{a c}-\frac{i}{2}\left(\Omega_{p}\left(2 \rho_{a a}+\rho_{b b}-1\right)-\Omega_{l} \sigma_{c b}^{*}\right) \\
\dot{\sigma}_{c b}=-\left(\gamma_{3}+i\left(\Delta_{l}-\Delta_{p}\right)\right) \sigma_{c b}-\frac{i}{2}\left(\Omega_{l} \sigma_{a c}^{*}-\Omega_{p} \sigma_{a b}\right),
\end{array}\right.
$$

with $R_{a}=-0.505 \rho_{a a}-0.405 \rho_{b b}+0.45, R_{b}=0.0495 \rho_{a a}-0.0505 \rho_{b b}+0.0055$ and $\Delta_{l}=\Delta_{c a v}+g \Re\left(\sigma_{a b}\right) \Omega_{l}$. The fixed parameters are $\gamma_{1}=0.275, \gamma_{2}=$ $0.25525, \gamma_{3}=0.25025, \gamma_{c a v}=0.03, g=100, \Delta_{p}=0$. The parameters $\Omega_{p}$ and $\Delta_{\text {cav }}$ are varied. The bifurcation diagram of (80) is computed in [26] and is reproduced in Figure 5 to facilitate reading. 


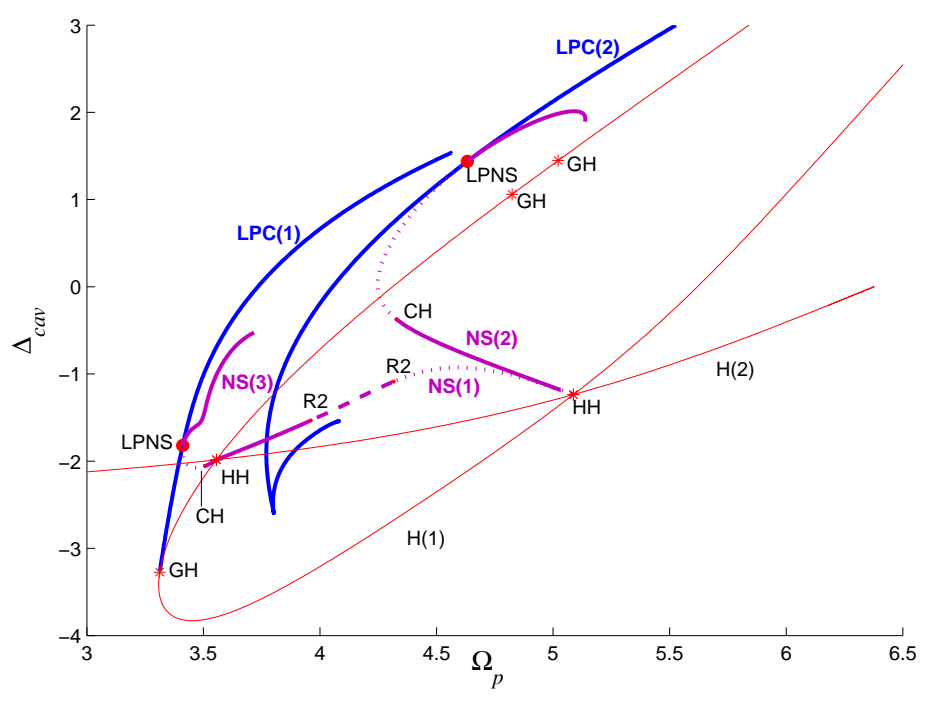

Figure 5: Bifurcation diagram of (80). The thin red curves are Hopf curves. In blue are limit point of cycles bifurcations and in magenta Neimark-Sacker bifurcations. Solid/dotted curves correspond to supercritical/subcritical bifurcations. The dashed curves are curves of neutral saddles.

\subsubsection{The LPNS points}

Figure 5 shows three NS curves NS(1), NS(2) and NS(3) starting from two $\mathrm{HH}$ points. On NS(3) one of the richer situations happens. The normal form coefficients for the LPNS point at $\left(\Omega_{p}, \Delta_{\text {cav }}\right)=(3.411,-1.819)$ are $(s, \theta, E)=(1,-0.139,-911.248)$, so $s \theta<0$. This means that there exists a 3 -torus, which is stable since $\theta<0$ and $E<0$. Therefore, we are in the case represented in Figure 1 (c), but with a stable 3-torus. For computing the Lyapunov exponents, we used a code written by V. N. Govorukhin (2004). Figure 6 (left) shows the Lyapunov exponents for $\Omega_{p}$ fixed at 3.45 and $\Delta_{\text {cav }} \in[-1.8 ;-1.6]$. More detail is shown in Figure 6 (right), where we get a clear view on the number of Lyapunov exponents equal to zero. For $\Delta_{\text {cav }}$ values to the right of -1.636 , there is one Lyapunov exponent equal to zero, which corresponds to the stable limit cycle from region 6 in Figure $1(\mathrm{c})$. At $\Delta_{\text {cav }}=-1.636$, we cross $\mathrm{NS}(3)$ and arrive in region 5 with a stable 2-torus and therefore two Lyapunov exponents equal to zero. When crossing the heteroclinic curve at point $P$ at $\Delta_{\text {cav }}=-1.773$, the stable 3torus from region 4 arises. Remark that in some small intervals only two 
Lyapunov exponents are equal to zero, and thus not the expected three zero ones, but these correspond with resonances on the 3 -torus. Then, in the interval $\Delta_{\text {cav }} \in[-1.796 ;-1.7916]$ positive Lyapunov exponents appear which indicate that there is chaos. This zone is delimited by the points indicated with $T$. Afterwards, we arrive in region 3, where all Lyapunov exponents are negative.
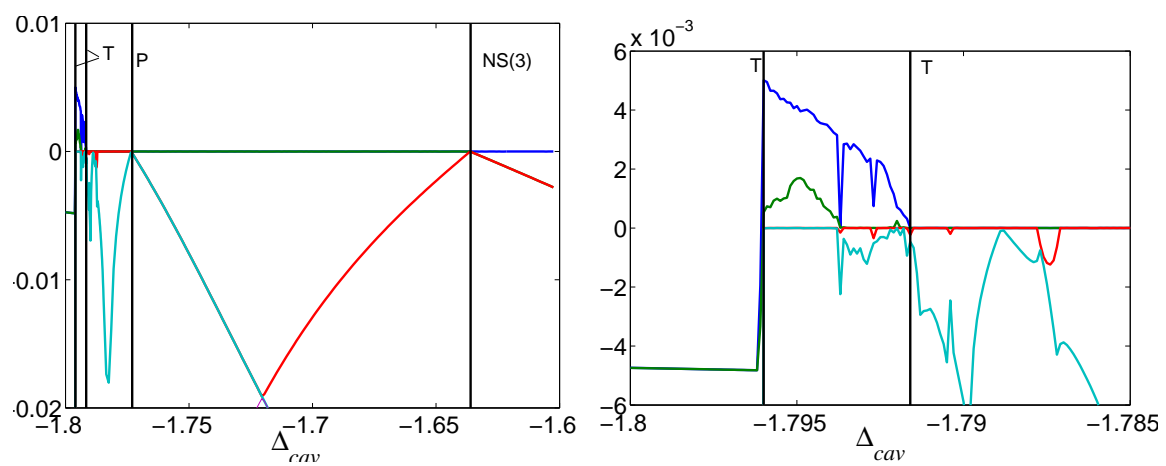

Figure 6: Lyapunov exponents computed for $\Omega_{p}=3.45$ close to the LPNS point at $\left(\Omega_{p}, \Delta_{\text {cav }}\right)=(3.411,-1.819)$, (left) for $\Delta_{\text {cav }} \in[-1.8 ;-1.6]$ and (right) zoomed in near the region with chaos due to heteroclinic tangles. The vertical black lines indicate the parameter values where a bifurcation occurs.

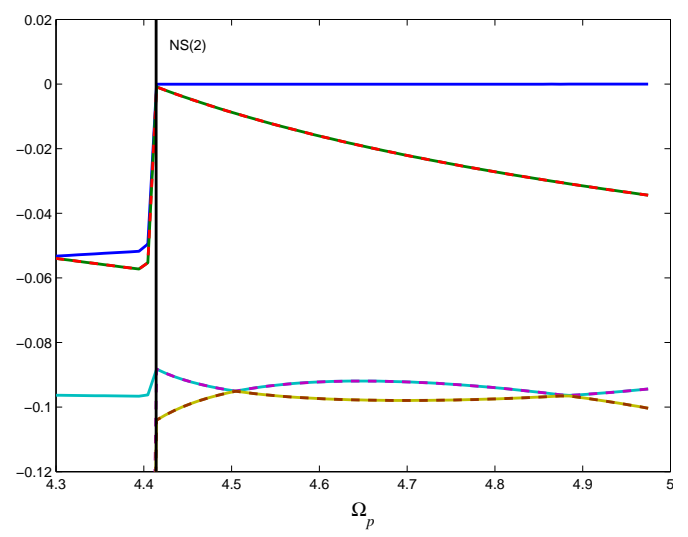

Figure 7: Lyapunov exponents computed close to the LPNS point at $\left(\Omega_{p}, \Delta_{\text {cav }}\right)=$ $(4.632,1.438)$. The two-coloured dashed lines reveal pairs of equally large Lyapunov exponents.

On the NS(2) curve there is one LPNS point for $\left(\Omega_{p}, \Delta_{\text {cav }}\right)=(4.632,1.438)$. The normal form coefficients are $(s, \theta, E)=(1,0.206,808.009)$. The product 
$s \theta>0$ is positive, so we are in a "simple" case, where no 3 -torus is present. Since $s=1$, the torus arisen through the Neimark-Sacker curve exists below the NS(2) curve. We have computed the Lyapunov exponents for a straight line where the beginning point $\left(\Omega_{p}, \Delta_{\text {cav }}\right)=(4.302,0.673)$ and end point $\left(\Omega_{p}, \Delta_{\text {cav }}\right)=(4.984,1.984)$ lie between the curves LPC(2) and NS(2), to the left and to the right of the LPNS point. In Figure 7, we plot the Lyapunov exponents for $\Omega_{p} \in[4.3,4.98]$. The stable limit cycle is situated in the upper wedge between the LPC(2) and NS(2) curves which corresponds to region 4 in Figure 1 (a), so we have one Lyapunov exponent equal to zero for $\Omega_{p^{-}}$ values larger than the subcritical NS(2) curve. At $\Omega_{p} \approx 4.41$, we cross the subcritical NS(2) curve, with to the left only negative Lyapunov exponents as the orbit went to an equilibrium.

\subsection{Periodic predator-prey model}

As a second model we study a simple two-patch predator-prey system with periodic (seasonal) forcing. Simple predator-prey models lead to the 'paradox of enrichment', i.e., increasing the carrying capacity of the prey ultimately leads to extinction of the population [27]. Outside the laboratory, however, stable populations are observed and not extinction. Here, spatial models have been put forward to explain this discrepancy. As the simplest spatial case, one may consider a two-patch predator-prey model [28] where predator and prey can migrate between the two patches by diffusion. This leads to a diffusive instability of large oscillations and stabilizes the total population size [29]. Here, we propose an extension where one of the patches experiences seasonal influences while the other can be seen as a wild-life refuge where human intervention minimizes seasonal influences. As a simplication we will only consider the case that the predators can move between the patches, i.e. they can cross the refuge barrier. On a proper time scale, the investigated system is defined by

$$
\left\{\begin{array}{l}
\dot{x}_{1}=r_{1} x_{1}\left(1-x_{1}\right)-\frac{c x_{1} x_{2}}{x_{1}+b_{1}\left(1+\varepsilon v_{1}\right)}, \\
\dot{x}_{2}=-x_{2}+\frac{c x_{1} x_{2}}{x_{1}+b_{1}\left(1+\varepsilon v_{1}\right)}+\gamma\left(y_{2}-x_{2}\right), \\
\dot{y}_{1}=r_{2} y_{1}\left(1-y_{1}\right)-\frac{c y_{1} y_{2}}{y_{1}+b_{2}} \\
\dot{y}_{2}=-y_{2}+\frac{c y_{1} y_{2}}{y_{1}+b_{2}}+\gamma\left(x_{2}-y_{2}\right), \\
\dot{v}_{1}=-v_{2}+v_{1}\left(1-v_{1}^{2}-v_{2}^{2}\right) \\
\dot{v}_{2}=v_{1}+v_{2}\left(1-v_{1}^{2}-v_{2}^{2}\right) .
\end{array}\right.
$$


The values of $x_{1}$ and $x_{2}$ denote the numbers of individuals (or densities) respectively of prey and predator populations living outside the refuge and $y_{1}$ and $y_{2}$ are the corresponding numbers or densities inside. The intrinsic growth rates $r_{i}$ and the constant attack rate $c$ are parameters of the model. For the predator outside the refuge, the Holling type II is chosen as functional response with a half saturation which varies periodically with period $2 \pi$. To this end, the last two equations are introduced; their solutions converge to a stable limit cycle $v_{1}(t)=\cos (t+\phi)$ with a phase shift $\phi$ depending on the initial conditions. The terms with parameter $\gamma$ describe the coupling of the two patches. The fixed parameter values are $r_{1}=1, r_{2}=1, b_{1}=0.4, \gamma=$ $0.1, c=2$. We will use the half saturation $b_{2}$ as a continuation parameter together with the amplitude of the seasonal forcing $\varepsilon$. It is not our aim to fully study this model, but rather analyze the codim 2 bifurcations relevant for this paper. We observe that a refuge can induce complex behaviour in a spatial population model with seasonal forcing.

\subsubsection{The PDNS points}

Figure 8 represents a bifurcation diagram for system (81) where two PDNS points are found. The right PDNS point has parameter values $\left(b_{2}, \varepsilon\right)=$ $(0.277,0.530)$. We are in the "simple" case of Section 2.2.2 because the product of the coefficients $p_{11}=-5.01 \cdot 10^{-2}$ and $p_{22}=-0.211$ is positive. Since $\theta=-0.320$ and $\delta=1.087$, Figure 1 (a) in indicates that the bifurcation diagram in a neighbourhood of the PDNS point is as in case III in Figure $3(\mathrm{a})$, where $\mu_{1}=0$ corresponds with NS1 and $\mu_{2}=0$ with PD. Curve $T_{1}$ corresponds to the Neimark-Sacker curve of the period doubled cycle NS2(2) in Figure 8. Therefore, we expect the period doubling 'curve' T2 of the torus to be situated to the left of NS1(2) and under the PD curve. The stable limit cycles are situated in the lower right region of the PDNS point. The exact location of T2 can be determined by computing Lyapunov exponents for fixed $b_{2}$ values smaller than the critical $b_{2}=0.277$ corresponding with the PDNS point. We have plotted a sketch of this T2 curve in Figure 9 (a), which represents a zoom of the neighbourhood of the PDNS point and which includes a plot of NS2(2) (curve $T_{1}$ in Figure 3 (a). We have computed the Lyapunov exponents for $b_{2}$ fixed at 0.261 and $\varepsilon \in[0.46 ; 0.62]$, see Figure 9 (b). In this figure the black vertical lines indicate the position of the PD and NS2(2) curves. From the value of the Lyapunov exponents we derive that T2 is crossed for $\varepsilon \approx 0.52$. To the left of the T2 curve in Figure 9 (b), we have a stable torus, arisen through the supercritical Neimark-Sacker 


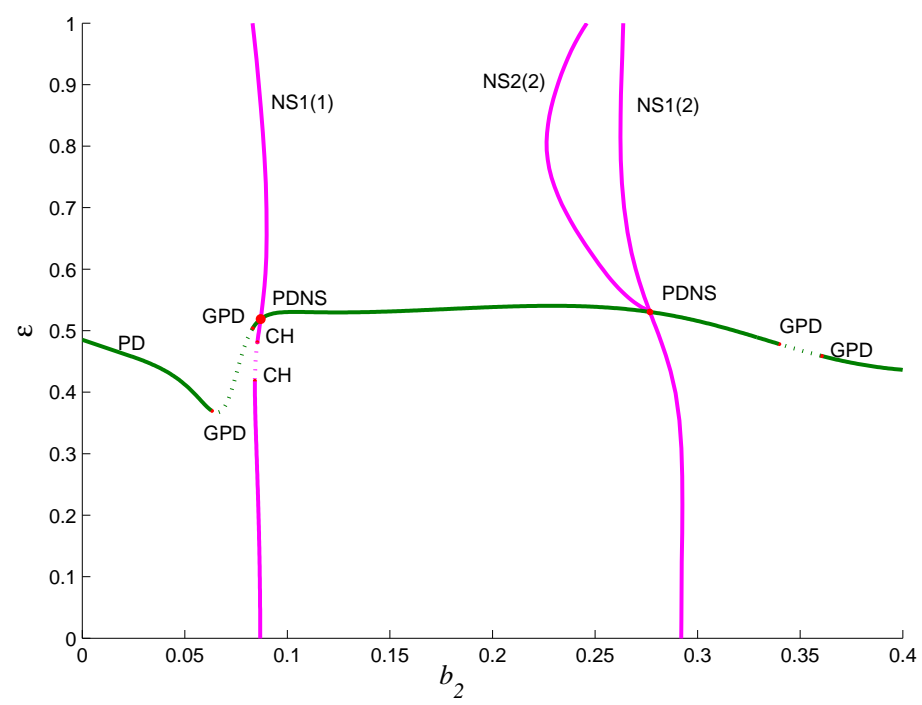

Figure 8: Bifurcation diagram of limit cycles in (81). In green are period doubling curves and in magenta Neimark-Sacker curves (of the first or of the second iterate, respectively labeled with NS1 and NS2).

curve NS1(2), corresponding with region 2 from Figure 3(b). Between the curves T2 and NS2(2), the 2-torus arisen through T2 is attracting. These regions correspond with region 6 (between T2 and PD) and 5 (between PD and NS2(2)) from Figure 3 (b). When crossing the NS2(2) curve, the 2-torus disappears and the period doubled cycle becomes attracting. All this is in agreement with the fact that two Lyapunov exponents are equal to zero to the left of NS2(2), where afterwards only one zero Lyapunov exponent is left.

The left PDNS point at $\left(b_{2}, \varepsilon\right)=\left(8.699 \cdot 10^{-2}, 0.519\right)$ again belongs to one of the "simple" situations in Section $2.2 .2\left(p_{11}=-0.447, p_{22}=-1.472\right)$. The neighbourhood of the bifurcation point is as in case I in Figure 1 (a) since $(\theta, \delta)=(2.234,1.304)$. Remark that the stable limit cycles are situated in the lower left quadrant of the PDNS point in Figure 10 (a). The behaviour in a neighbourhood of this PDNS point can be derived from Figure 10 (a), which includes a plot of the Neimark-Sacker curve NS2(1) of the period doubled cycle and also a sketch of the period doubled curve T2 of the torus, made on the basis of the computation of the Lyapunov exponents. We have calculated the Lyapunov exponents for parameter values in the upper right 

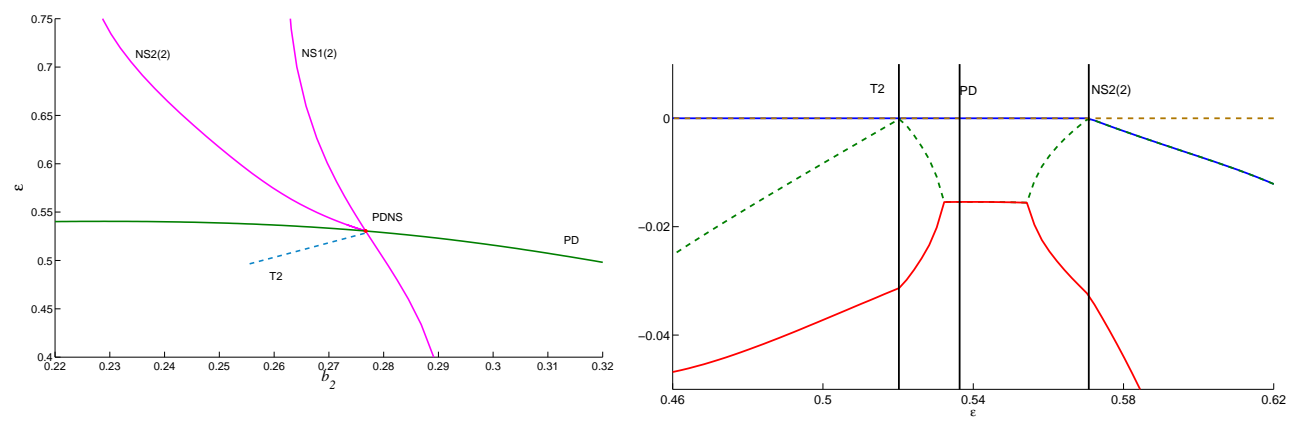

Figure 9: (a) Zoom of the neighbourhood of the PDNS point at $\left(b_{2}, \varepsilon\right)=(0.277,0.530)$ from Figure 8. In blue is the sketch of the T2 'curve'. (b) Lyapunov exponents computed for $b_{2}=0.261$, close to the PDNS point at $\left(b_{2}, \varepsilon\right)=(0.277,0.530)$.
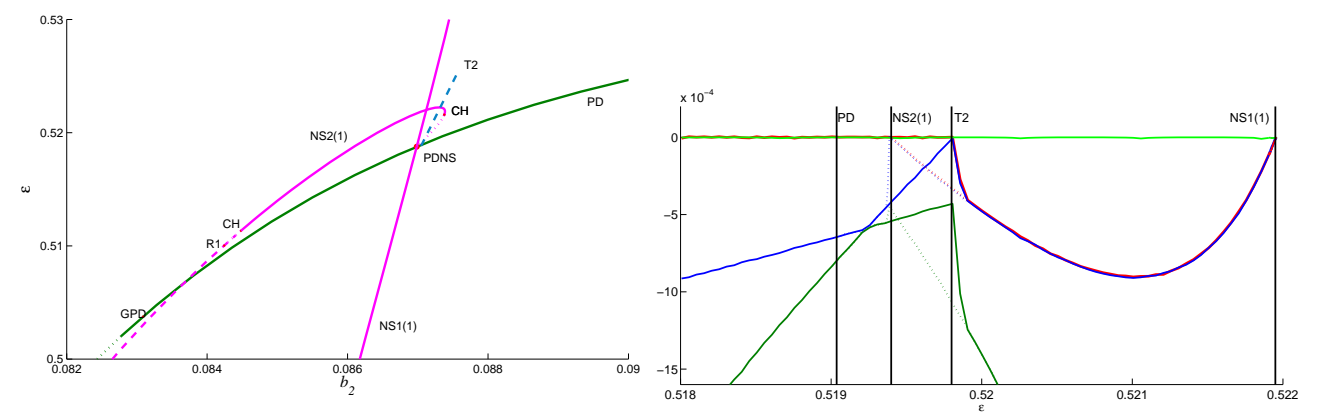

Figure 10: (a) Zoom of the neighbourhood of the PDNS point at $\left(b_{2}, \varepsilon\right)=(8.699$. $10^{-2}, 0.519$ ) from Figure 8. In blue is the sketch of the T2 'curve'. (b) Lyapunov exponents computed for $b_{2}=0.08709$, close to the PDNS point at $\left(b_{2}, \varepsilon\right)=\left(8.699 \cdot 10^{-2}, 0.519\right)$. Exponents indicated with solid lines are computed by following the attractor with increasing $\varepsilon$, dotted lines with decreasing $\varepsilon$. This highlights the bistability between NS2(1) and T2.

quadrant, close to the PDNS point, for $b_{2}=0.08709$. The results are given in Figure 10 (b). Going from the left to the right, where we follow the solid lines, we start with two Lyapunov exponents equal to zero which correspond with the stable torus from the original cycle in the regions 2,3 and 4 from Figure 3. At the point where the second Lyapunov exponent becomes nonzero, the T2 curve is located, namely at $\varepsilon \approx 0.5198$. We then arrive in region 12 from Figure 3 (b) where the 2-torus has lost his stability and the period doubled cycle is stable. Therefore, one zero Lyapunov exponent remains. We scan the Lyapunov exponents for a second time where we now go from the 
right to the left and follow the dashed lines. The second Lyapunov exponent now approaches zero not at the T2 curve but at the NS2(1) curve. This is explained by the bistability happening in region 4, where one Lyapunov exponent equal to zero indicates the stable period doubled cycle and two zero Lyapunov exponents indicate the stable torus. When going further, we cross region 3 and 2, with the stable torus of the orginal cycle.

Remark that since we have a periodically forced system the return time is independent of the distance from the limit cycle, so we could do this extra check. Indeed, for all PDNS points, the $\alpha_{i j k}$ in the first equation of (5) are zero up to the accuracy of the computation. Here too, the Lyapunov exponents corroborate the prediction based on the normal form coefficients.

\subsection{Control of vibrations}

In [30] a two-mass system of which the main mass is excited by a flowinduced, self excited force is studied. A single mass which acts as a dynamic absorber is attached to the main mass and, by varying the stiffness between the main mass and the absorber mass, represents a parametric excitation. The system is given by

$$
\left\{\begin{array}{l}
\dot{x}_{1}=v_{1} \\
\dot{x}_{2}=v_{2} \\
\dot{v}_{1}=-k_{1}\left(v_{1}-v_{2}\right)-Q^{2}\left(1+\varepsilon y_{1}\right)\left(x_{1}-x_{2}\right) \\
\dot{v}_{2}=M k_{1}\left(v_{1}-v_{2}\right)+M Q^{2}\left(1+\varepsilon y_{1}\right)\left(x_{1}-x_{2}\right)-k_{2} v_{2}-x_{2}+\beta V^{2}\left(1-\gamma v_{2}^{2}\right) v_{2} \\
\dot{y}_{1}=-\eta y_{2}+y_{1}\left(1-y_{1}^{2}-y_{2}^{2}\right) \\
\dot{y}_{2}=\eta y_{1}+y_{2}\left(1-y_{1}^{2}-y_{2}^{2}\right) .
\end{array}\right.
$$

The following parameters are fixed: $\varepsilon=0.1, k_{2}=0.1, \beta=0.1, V=\sqrt{2.1}, \gamma=$ 4, $Q=0.95, M=0.2, k_{1}$ and $\eta$ will be the continuation parameters.

\subsubsection{The NSNS points}

An NSNS point is detected for $\left(k_{1}, \eta\right)=\left(9.167 \cdot 10^{-2}, 0.411\right)$, see Figure 11. The normal form coefficients are

$$
\left(p_{11}, p_{22}, \theta, \delta, \operatorname{sign} l_{1}\right)=\left(-3.733 \cdot 10^{-3},-6.494 \cdot 10^{-3}, 0.541,1.203,1\right) .
$$

The positive sign of the product $p_{11} p_{22}$ implies that we are in a "simple" case of Section 2.2.3. Since $\delta>\theta$, the role of both coefficients has to be 


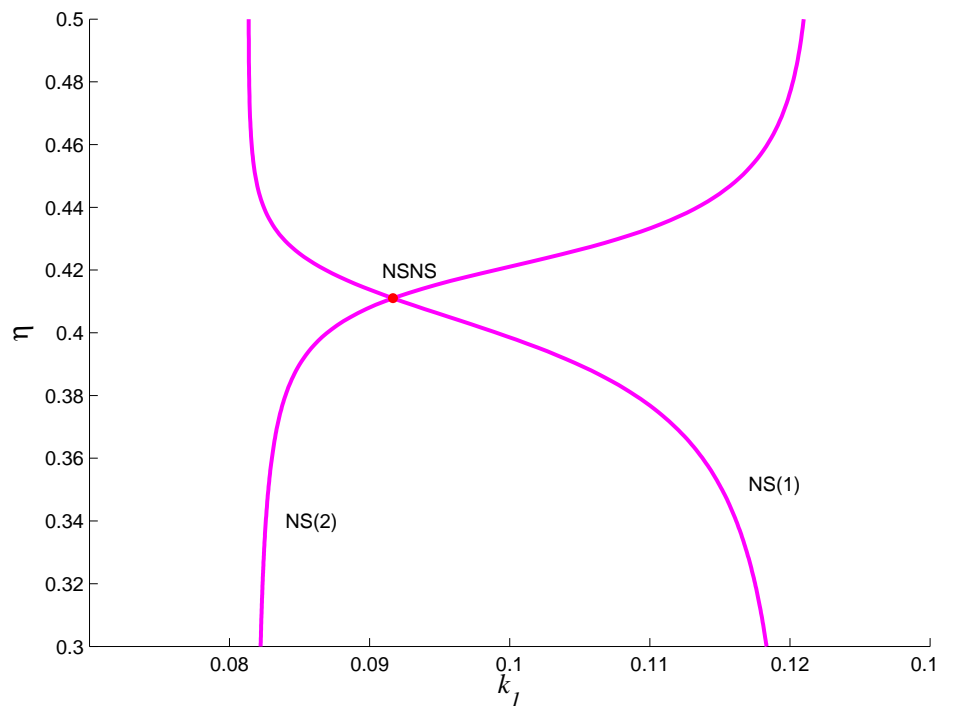

Figure 11: Partial bifurcation diagram of limit cycles in system (82).

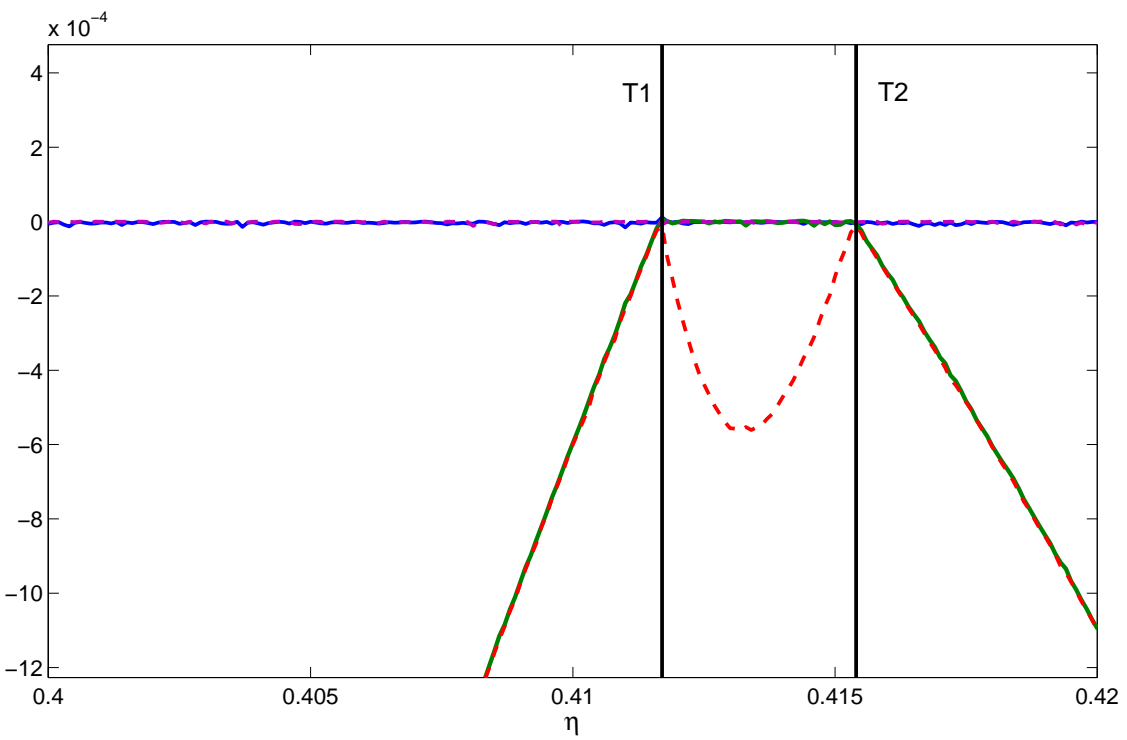

Figure 12: Lyapunov exponents computed for $k_{1}=0.083$. 
reversed. Therefore, $\theta>1, \delta<1, \theta \delta<1$ indicate that the NSNS bifurcation is located in region II in Figure 3 (a). As in the previous examples, we have computed the Lyapunov exponents to check the obtained results of the normal form coefficients. We have done the computations for $k_{1}$ fixed at 0.083 and $\eta \in[0.4 ; 0.42]$ ( $\eta$ values are between the NS curves). The results are given in Figure 12. For $\eta$-values starting from 0.38 , we are in region 3 (or 12 due to symmetry) in Figure 3(b), where there is a stable 2-torus and thus two Lyapunov exponents equal to zero. A third Lyapunov exponent approaches zero and between $\eta \approx 0.4117$ and $\eta \approx 0.4154$ three Lyapunov exponents are equal to zero. This region denotes the appearance of a stable 3 -torus and corresponds with region 5 from Figure 3 (b). The critical values of $\eta$ correspond with the curves $T_{1}$ and $T_{2}$ in Figure 3(a). For $\eta \geq 0.4154$, only a stable 2-torus remains such that there are two zero Lyapunov exponents. Therefore, the computed Lyapunov exponents are in agreement with the normal form coefficients.

Also in this case all $\alpha_{i j k l}$ in the normal form (6) vanish since we have a periodically forced system.

\section{Discussion}

This paper completes the development of efficient methods for the computation of the critical normal form coefficients for all codim 1 and 2 local bifurcations of limit cycles, started in [16, 17] and based on [14]. Together with our previous papers on the computation of the critical normal form coeffcients for codim 1 and 2 local bifurcations of equilibria in ODEs [31] and fixed points of maps $[13,8]$, it contributes to the development of methods, algorithms, and software tools for multiparameter bifurcation analysis of smooth finite-dimensional dynamical systems.

The resulting formulas are independent of the phase space dimension and can be applied in the original basis, without preliminary linear transformations. As limit cycles are concerned, the formulas are directly suitable for numerical implementation using orthogonal collocation. They fit perfectly into a continuation context, where limit cycles and their bifurcations are computed using the BVP-approach [32], without numerical approximation of the Poincaré map or its derivatives. Being implemented into the MATLAB toolbox MATCONT $[2,3]$, the methods developed are freely available to assist an advanced two-parameter bifurcation analysis of dynamical systems generated by ODEs and maps from various applications. 
In the present paper we deal with the three most complex cases, LPNS, PDNS and NSNS, in which bifurcations of tori play an essential role. We heavily rely on the computation of Lyapunov exponents to provide evidence for the existence of bifurcations of tori.

To fully support the two-parameter bifurcation analysis of ODEs and maps, one needs special methods to switch between various branches of codim 1 bifurcations of fixed points and cycles rooted at codim 2 points. Such methods have been developed and implemented in MATCONT for codim 2 equilibrium [26] and fixed point [9] bifurcations. Switching at codim 2 points to the continuation of codim 1 local bifurcations of limit cycles seems to be the next natural problem to attack, while that for codim 1 bifurcations of homoclinic and heteroclinic orbits is more difficult and probably requires

new computational ideas. Similar remarks can be made about quasiperiodic bifurcations of tori.

\section{Appendix A. Bifurcations of the amplitude system for Hopf-Hopf bifurcation in the "difficult" case}

Here, we derive quadratic approximations of the Hopf and heteroclinic bifurcation curves for the double Hopf amplitude system (10) that in this case can be reduced to the form

$$
\left(\begin{array}{c}
\dot{x} \\
\dot{y}
\end{array}\right)=\left(\begin{array}{l}
x\left(\mu_{1}+x-\theta y+\Theta y^{2}\right) \\
y\left(\mu_{2}+\delta x-y+\Delta x^{2}\right)
\end{array}\right),
$$

where $\theta, \delta, \Theta$, and $\Delta$ are defined by $(11)$.

The main results are

$$
\begin{aligned}
\mu_{1, C} & =-\frac{\theta-1}{\delta-1} \mu_{2}-\frac{(\delta-1) \Theta+(\theta-1) \Delta}{(\delta-1)^{3}} \mu_{2}^{2}, \\
\mu_{1, Y} & =-\frac{\theta-1}{\delta-1} \mu_{2}+\frac{\theta \Theta(\delta-1)^{3}+\delta \Delta(\theta-1)^{3}}{(\delta-1)^{3}(2 \delta \theta-\delta-\theta)} \mu_{2}^{2}, \\
l_{1} & =-\delta(\delta(\delta-1) \Theta+\theta(\theta-1) \Delta) .
\end{aligned}
$$

For the Hopf bifurcation curve $C$ we impose the conditions $\dot{x}=0, \dot{y}=$ 0 and $\frac{\partial \dot{x}}{\partial x}+\frac{\partial \dot{y}}{\partial y}=0$. Solving a series expansion yields the result for the curve. Next, the first Lyapunov coefficient $l_{1}$ is computed using the invariant formula. 
For the heteroclinic curve $Y$ we proceed as follows. We assume $\delta, \theta<0$ and $\delta \theta-1>0$ and we transform variables to obtain a system that is a perturbation of a Hamilton-system. This enables to formulate a Melnikov function. Setting this function to zero yields an equation from which we extract the quadratic approximation to the heteroclinic curve. Introducing the transformation $\left(t, x, y, \mu_{1}, \mu_{2}\right) \rightarrow\left(\varepsilon x^{p-1} y^{q-1} t, \varepsilon x, \varepsilon y, c_{1} \varepsilon+c_{2} \varepsilon^{2}, \varepsilon\right)$ where

$$
c_{1}=-\frac{\theta-1}{\delta-1}, p=\frac{1-\delta}{\delta \theta-1}, q=\frac{1-\theta}{\delta \theta-1} .
$$

Then we obtain

$$
\left(\begin{array}{c}
\dot{x} \\
\dot{y}
\end{array}\right)=x^{p-1} y^{q-1}\left(\begin{array}{c}
x\left(c_{1}+x-\theta y\right) \\
y(1+\delta x-y)
\end{array}\right)+\varepsilon x^{p-1} y^{q-1}\left(\begin{array}{c}
c_{2} x+\Theta x y^{2} \\
\Delta y x^{2}
\end{array}\right),
$$

which for $\epsilon=0$ is a Hamilton system with Hamiltonian

$$
H(x, y)=\frac{1}{p} x^{p} y^{q}\left(-1+\frac{\delta-1}{\theta-1} x+y\right) .
$$

The Melnikov function along the nontrivial critical curve $H(x, y)=0$ is given by the following integral

$$
\begin{aligned}
M(h) & =\int_{H=h} g_{1} d y-g_{2} d x \\
& =\int_{H=h} x^{p} y^{q-1}\left(c_{2}+\Theta y^{2}\right) d y-\Delta x^{p+1} y^{q} d x \\
& =\int_{H=h}\left(x^{p} y^{q-1}\left(c_{2}+\Theta y^{2}\right)+\frac{q \Delta}{p+2} x^{p+2} y^{q-1}\right) d y
\end{aligned}
$$

where we used Green's Theorem to convert the $d x$ term to $d y$. Now along the nontrivial critical curve $H(x, y)=0$ we have $x=\frac{\theta-1}{\delta-1}(1-y)$ so that

$$
\begin{aligned}
M(0) & =\left(\frac{\theta-1}{\delta-1}\right)^{p} \int_{0}^{1}(1-y)^{p} y^{q-1}\left(c_{2}+\Theta y^{2}+\left(\frac{\theta-1}{\delta-1}\right)^{2} \frac{q \Delta}{p+2}(1-y)^{2}\right) d y \\
& \sim c_{2} I_{p, q-1}+\Theta I_{p, q+1}+\left(\frac{\theta-1}{\delta-1}\right)^{2} \frac{q \Delta}{p+2} I_{p+2, q-1}
\end{aligned}
$$

where we defined

$$
I_{a, b}=\int_{0}^{1}(1-y)^{a} y^{b} d y=\frac{\Gamma(1+a) \Gamma(1+b)}{\Gamma(2+a+b)} .
$$


Solving $M(0)=0$ and substituting $p, q$ we obtain

$$
c_{2}=\frac{\theta \Theta(\delta-1)^{3}-\delta \Delta(1-\theta)^{3}}{(\delta-1)^{3}(2 \delta \theta-\delta-\theta)} .
$$

As final check we consider the difference between the quadratic approximations of the heteroclinic and the Hopf curves

$$
\mu_{1, Y}-\mu_{1, C}=-\frac{(\delta \theta-1) l_{1}}{\delta(\delta-1)^{3}(2 \delta \theta-\delta-\theta)} \mu_{2}^{2}
$$

We see that these approximations coincide precisely when the Hopf bifurcation is degenerate, i.e. $l_{1}=0$.

\section{References}

[1] E. J. Doedel, A. R. Champneys, T. F. Fairgrieve, Yu. A. Kuznetsov, B. Sandstede, X. J. Wang, Auto97: Continuation and bifurcation software for ordinary differential equations (with HomCont) (1997).

[2] A. Dhooge, W. Govaerts, Yu. A. Kuznetsov, MATCONT: A MATLAB package for numerical bifurcation analysis of ODEs, ACM Trans. Math. Software 29 (2) (2003) 141-164.

[3] A. Dhooge, W. Govaerts, Y. A. Kuznetsov, H. G. E. Meijer, B. Sautois, New features of the software MatCont for bifurcation analysis of dynamical systems, Math. Comput. Model. Dyn. Syst. 14 (2) (2008) 147-175.

[4] V. I. Arnol'd, Geometrical Methods in the Theory of Ordinary Differential Equations, Springer-Verlag, New York, 1983.

[5] G. Iooss, Bifurcation of Maps and Applications, Vol. 36 of North-Holland Mathematics Studies, North-Holland Pub. Co., Amsterdam, 1979.

[6] J. Guckenheimer, Ph. Holmes, Nonlinear Oscillations, Dynamical Systems and Bifurcations of Vector Fields, Springer-Verlag, New York, 1983.

[7] Yu. A. Kuznetsov, Elements of Applied Bifurcation Theory, SpringerVerlag, New York, 2004, 3rd ed. 
[8] Yu. A. Kuznetsov, H. G. E. Meijer, Remarks on interacting Neimark-Sacker bifurcations, J. Difference Equ. Appl. 12 (10) (2006) 1009-1035.

[9] W. Govaerts, R. Khoshsiar Ghaziani, Yu. A. Kuznetsov, H. G. E. Meijer, Numerical methods for two-parameter local bifurcation analysis of maps, SIAM J. Sci. Comput. 29 (6) (2007) 2644-2667.

[10] R. Vitolo, H. Broer, C. Simó, Routes to chaos in the Hopf-saddle-node bifurcation for fixed points of 3D-diffeomorphisms, Nonlinearity 23 (8) (2010) 1919-1948.

[11] C. Simó, Analytical and numerical computation of invariant manifolds, in: C. Benest, C. Froeschlé (Eds.), Modern Methods in Celestial Mechanics, Editions Frontiéres, 1990, pp. 285-330.

[12] J. Guckenheimer, B. Meloon, Computing periodic orbits and their bifurcations with automatic differentiation, SIAM J. Sci. Comput. 22 (3) (2000) 951-985.

[13] Yu. A. Kuznetsov, H. G. E. Meijer, Numerical normal forms for codim 2 bifurcations of fixed points with at most two critical eigenvalues, SIAM J. Sci. Comput. 26 (6) (2005) 1932-1954.

[14] G. Iooss, Global characterization of the normal form for a vector field near a closed orbit, J. Differential Equations 76 (1) (1988) 47-76.

[15] G. Iooss, M. Adelmeyer, Topics in Bifurcation Theory and Applications, Vol. 3 of Advanced Series in Nonlinear Dynamics, World Sci. Pub. Co. Inc., River Edge, New York, 1992.

[16] Yu. A. Kuznetsov, W. Govaerts, E. J. Doedel, A. Dhooge, Numerical periodic normalization for codim 1 bifurcations of limit cycles, SIAM J. Numer. Anal. 43 (4) (2005) 1407-1435.

[17] V. De Witte, F. Della Rossa, W. Govaerts, Yu. Kuznetsov, Numerical periodic normalization for codim 2 bifurcations of limit cycles - computational formulas, numerical implementation, and examples, SIAM J. Appl. Dyn. Systems 12 (2) (2013) 722-788. 
[18] V. De Witte, W. Govaerts, Y. A. Kuznetsov, H. G. E. Meijer, Numerical periodic normalization for codim 2 bifurcations of limit cycles with center manifold of dimension higher than 3 . http://arxiv.org/abs/1210.6205.

[19] V. Arnol'd, V. Afrajmovich, Yu. Il'yashenko, L. Shil'nikov, Dynamical Systems. V, Vol. 5 of Encyclopaedia of Mathematical Sciences, SpringerVerlag, Berlin, 1994, Bifurcation Theory and Catastrophe Theory, A translation of "Current problems in mathematics. Fundamental directions". Vol. 5 (Russian), Akad. Nauk SSSR, Vsesoyuz. Inst. Nauchn. i Tekhn. Inform., Moscow, 1986. Translation by N. D. Kazarinoff.

[20] J. Los, Nonnormally hyperbolic invariant curves for maps in r 3 and doubling bifurcation, Nonlinearity 2 (1) (1989) 149.

[21] R. Vitolo, H. Broer, C. Simó, Quasi-periodic bifurcations of invariant circles in low-dimensional dissipative dynamical systems, Regul. Chaotic Dyn. 16 (1-2) (2011) 154-184.

[22] Yu. A. Kuznetsov, V. V. Levitin, CONTENT: A multiplatform environment for analyzing dynamical systems, Dynamical Systems Laboratory, CWI, Amsterdam (1997).

[23] C. De Boor, B. Swartz, Collocation at Gaussian points, SIAM J. Numer. Anal. 10 (4) (1973) 582-606.

[24] U. M. Ascher, R. M. M. Mattheij, R. D. Russell, Numerical solution of boundary value problems for ordinary differential equations, Vol. 13 of Classics in Applied Mathematics, Society for Industrial and Applied Mathematics (SIAM), Philadelphia, PA, 1995, corrected reprint of the 1988 original.

[25] S. Wieczorek, W. W. Chow, Self-induced chaos in a single-mode inversionless laser, Phys. Rev. Lett. 97.

[26] Yu. A. Kuznetsov, H. G. E. Meijer, W. Govaerts, B. Sautois, Switching to nonhyperbolic cycles from codim 2 bifurcations of equilibria in ODEs, Phys. D 237 (23) (2008) 3061-3068.

[27] M. Rosenzweig, Paradox of enrichment: Destabilization of exploitation ecosystems in ecological time, Science 171 (3969) (1971) 385-387. 
[28] V. Jansen, Regulation of predator-prey systems through spatial interactions: A possible solution to the paradox of enrichment, Oikos 74 (3) (1995) 384-390.

[29] V. Jansen, The dynamics of two diffusively coupled predator-prey populations, Theoretical Population Biology 59 (2) (2001) 119 - 131.

[30] S. Fatimah, F. Verhulst, Suppressing flow-induced vibration by parametric excitation, Nonlinear Dynam. 31 (2003) 275-297.

[31] Yu. A. Kuznetsov, Numerical normalization techniques for all codim 2 bifurcations of equilibria in ODEs, SIAM J. Numer. Anal. 36 (4) (1999) 1104-1124.

[32] E. J. Doedel, W. Govaerts, Yu. A. Kuznetsov, Computation of periodic solution bifurcations in ODEs using bordered systems, SIAM J. Numer. Anal. 41 (2) (2003) 401-435. 